\title{
A MegaCam Survey of Outer Halo Satellites. VII. A Single Sérsic Index versus Effective Radius Relation for Milky Way Outer Halo Satellites* ${ }^{*}$
}

\author{
Sebastián Marchi-Lasch ${ }^{1}$ (D), Ricardo R. Muñoz ${ }^{1}$, Felipe A. Santana ${ }^{1}$ (D), Julio A. Carballo-Bello ${ }^{2}$, Julio Chanamé2, Marla Geha ${ }^{3}$ (i), \\ Joshua D. Simon ${ }^{4}$, Peter B. Stetson ${ }^{5}$ (iD), and S. G. Djorgovski ${ }^{6}$ (i) \\ ${ }^{1}$ Departamento de Astronomía, Universidad de Chile, Camino el Observatorio 1515, Las Condes, Santiago, Chile; smarchi@das.uchile.cl \\ ${ }^{2}$ Instituto de Astrofísica, Facultad de Física, Pontificia Universidad Católica de Chile, Av. Vicuña Mackenna 4860, 782-0436 Macul, Santiago, Chile \\ 3 Astronomy Department, Yale University, New Haven, CT 06520, USA \\ ${ }^{4}$ Observatories of the Carnegie Institution for Science, 813 Santa Barbara Street, Pasadena, CA 91101, USA \\ ${ }^{5}$ National Research Council of Canada, Herzberg Astronomy and Astrophysics, 5071 W. Saanich Road, Victoria, BC V9E 2E7, Canada \\ ${ }^{6}$ Astronomy Department, California Institute of Technology, Pasadena, CA 91125, USA \\ Received 2018 November 8; revised 2019 February 14; accepted 2019 February 18; published 2019 March 19
}

\begin{abstract}
In this work, we use structural properties of the Milky Way's outer halo $\left(R_{G}>25 \mathrm{kpc}\right)$ satellites (dwarf spheroidal galaxies, ultra-faint dwarf (UFD) galaxies and globular clusters (GCs)) derived from deep, wide-field, and homogeneous data to present evidence of a correlation in the Sérsic index versus effective radius plane followed by a large fraction of outer halo GCs and satellite dwarf galaxies. We show that this correlation can be entirely reproduced by fitting empirical relations in the central surface brightness versus absolute magnitude and Sérsic index versus absolute magnitude parameter spaces, and by assuming the existence of two types of outer halo GCs: one of high surface brightness (HSB group), with properties similar to those of inner halo clusters; and another of low surface brightness (LSB group), which share characteristics with dwarf spheroidal and UFD galaxies. Given the similarities of LSB clusters with dwarf spheroidal and UFD galaxies, we discuss the possibility that outer halo clusters also originated inside dark matter halos and that tidal forces from different host galaxy potentials are responsible for the different properties between HSB and LSB clusters.
\end{abstract}

Key words: galaxies: dwarf - Galaxy: halo - globular clusters: general

\section{Introduction}

The halo of the Milky Way (MW) contains important information about the ancient history of our Galaxy, especially because dynamical scales are long enough to retain information of past Galactic events (e.g., Johnston et al. 1996; Mayer et al. 2002). A significant fraction of this information is contained in the structural, dynamical, and chemical properties of satellite stellar structures of the MW, which dominate the outer halo stellar distribution (see Majewski 2004; Willman 2010; Ivezić et al. 2012, for reviews on this topic). Thus, by studying these substructures, it is, in principle, possible to peer into our galaxy's past and learn about the processes that governed its formation and evolution.

The stellar structures that surround the Galaxy have been usually classified as either globular clusters (GCs) or dwarf galaxies. Specifically, most of the dwarf galaxies are of the dwarf spheroidal type (dSph), which is devoid of gas and shows no current stellar formation. Both types of stellar structures are dominated by an old, metal-poor stellar population. Currently, it is accepted that dwarf galaxies formed their own dark matter (DM) halos at small scales and were accreted later by the MW, as described by hierarchical growth models (Searle \& Zinn 1978; Bullock \& Johnston 2005). In the case of GCs, a fraction of them formed together with our galaxy during a phase of rapid collapse as proposed by Eggen et al. (1962), whereas others are thought to have an external

\footnotetext{
* Based on observations obtained at the Canada-France-Hawaii Telescope (CFHT), which is operated by the National Research Council of Canada, the Institut National des Sciences de l'Univers of the Centre National de la Recherche Scientifique of France, and the University of Hawaii.

$\dagger$ This paper includes data gathered with the $6.5 \mathrm{~m}$ Magellan Telescopes located at Las Campanas Observatory, Chile.
}

origin, i.e., they formed in galaxies that were later accreted by the MW, which stripped off their GCs (Zinn 1993, 1996; Mackey \& Gilmore 2004; Mackey \& van den Bergh 2005; Leaman et al. 2013; Zaritsky et al. 2016).

To understand better the role of these structures in the formation and evolution of the MW, current research efforts have focused on the detection of satellites in order to obtain a reliable census of satellite objects orbiting our Galaxy. The results have significantly changed the way we understand our Galaxy surroundings. Before 2005, only nine Galactic dSphs were known (now referred to as classical dSphs), with luminosities in the range $-12 \lesssim M_{\mathrm{V}} \lesssim-8$ and with half-light radii on the order of $100 \mathrm{pc}$. Regarding GCs, almost all of them were compact objects, with half-light radii of less than $10 \mathrm{pc}$ and, in general, less luminous than classical dSphs. Over the last decade and a half, and thanks to large area surveys like the Sloan Digital Sky Survey (SDSS; York et al. 2000), PanSTARRS1 (Chambers et al. 2016) and the Dark Energy Survey (DES; Dark Energy Survey Collaboration et al. 2016), the population of satellite systems has increased significantly, more than doubling the total number (Willman et al. 2005; Belokurov et al. 2006, 2007, 2008, 2009, 2010, 2014; Zucker et al. 2006; Walsh et al. 2007; Muñoz et al. 2012a; Bechtol et al. 2015; Kim et al. 2015; Koposov et al. 2015; Laevens et al. 2015a, 2015b; Martin et al. 2015; Torrealba et al. 2016a, 2016b; Homma et al. 2018). The new objects include low-luminosity dSphs $\left(M_{\mathrm{V}}>-8\right)$, named ultra-faint dwarf galaxies (UFDs), some of them as small as some GCs; and halo GCs, some of them of size comparable to these UFDs. In this new scenario, the size gap that seemed to separate GCs from $\mathrm{dSphs}$ in the size versus luminosity plot has started to become populated, casting doubts on the true different origins for 
extended GCs and UFDs (e.g., Drlica-Wagner et al. 2015; Torrealba et al. 2016a). For example, it is not clear whether the large half-light radii of extended GCs is an intrinsic property of a different class of objects or a result of interactions with the MW (e.g., van den Bergh \& Mackey 2004; Ripepi et al. 2007; Hwang et al. 2011).

One widely accepted difference between dSphs and GCs is in their DM content. For classical dSphs, the mass-to-light ratio within their half-light radii ranges from $\sim 6$ to $\sim 100$, and from $\sim 100$ to $\sim 3000$ for UFDs (see Figure 11 of McConnachie 2012), making the latter the most DM-dominated objects known in the universe. In the case of GCs, they have values consistent with no DM content, with typical values for the mass-to-light ratio of $\sim 1$ to $\sim 4$ (e.g., McLaughlin 2000; Rejkuba et al. 2007; Baumgardt et al. 2009). This feature, together with the different metallicity spread between dSphs and GCs, has become the standard for classifying a halo stellar overdensity either as a dSph or a GC (Willman \& Strader 2012). In principle, it is reasonable to think that the presence of DM should leave a distinct imprint in the structural and photometric parameters of the baryonic matter of dSphs, in stark contrast with GCs. To explore this idea and to shed some light into the different processes that formed these two types of substructures, it is useful to have a complete characterization of their respective structural and photometric properties and to compare them homogeneously.

This paper is part of a series of articles based on a catalog of structural parameters constructed from deep, wide, and homogeneous observations of 58 satellite objects located in the outer halo of the MW (Muñoz et al. 2018a, 2018b). These parameters include the half-light radius, surface brightness, luminosity, ellipticity, and Sérsic index. In P. Côté et al. (2019, in preparation), we study a wide range of scaling relations between the different objects in the catalog. In this article, we focus on the observed trend of the Sérsic index with effective radius, which shows a strong correlation when all outer halo objects are considered.

This article is organized as follows. In Section 2, we briefly describe our data set. In Section 3, we use the structural parameters derived from our data set to explore some properties of GCs and dSphs, while in Section 4 we concentrate our analysis on the Sérsic's index versus effective radius relation. Next, in Section 5 we provide an explanation for the origin of the previous correlation and discuss some consequences of its existence for the formation and evolution processes of GCs and dSphs. Finally, in Section 6, we present a summary and the conclusions of this work.

\section{Data}

The data set used in this work is composed of observations of 58 satellite objects of the MW, which includes GCs, classical dSphs, and UFDs and a number of objects not yet classified (i.e., their structural properties neither allow for a clear differentiation nor are their DM content or metallicity spread known). The classification for each object is based on information in the literature.

Observations of 44 of these objects were carried out using the MegaCam imager on the Canada-France-Hawaii Telescope (CFHT) in the northern hemisphere and the Megacam imager on the Magellan II-Clay Telescope at Las Campanas Observatory in the southern hemisphere. The data for the remaining 14 objects were obtained from different sources, most of them from public data from the DES Year Release 1 (see Muñoz et al. 2018a for details).

Muñoz et al. (2018a) described the data reduction, astrometry, point source photometry, and photometric calibration performed on the whole sample in order to obtain a homogeneous data set. To measure the structural parameters and density profiles, a maximum likelihood approach was applied to the observations of every object, assuming a Sérsic density profile (Sérsic 1968) plus a background density (Muñoz et al. 2018b). Absolute magnitude and surface brightness values were obtained by integrating a theoretical luminosity function for every object, which is normalized by the number of member stars of the object.

It is important to mention that, although our observations come from different instruments and full photometric homogeneity is not possible, as described in Muñoz et al. (2018a), care was taken to make the data set as homogeneous as possible: the Megacam imagers used to create the primary catalog are similar in structure and performance, the same bands were used for all 58 objects, the same reduction pipeline and techniques were used for all objects, and the spatial coverage for every object in our data set is comparable (at least five effective radii), with only a few exceptions.

The outer halo object data used in this work are presented in Table 1.

\section{Parameter Distributions}

Given the characteristics of our new data set (wider, deeper, and nearly homogeneous), in P. Côté et al. (2019, in preparation) we explore in depth a wide range of correlations between different structural parameters in order to globally assess the similarities and differences between GCs and dSphs. Here, we briefly highlight some of those results.

Figure 1 shows the distribution of six structural parameters: absolute magnitude in the $V$ band $\left(M_{\mathrm{V}}\right)$, effective radius $\left(R_{\mathrm{e}}\right)$, central surface brightness in the $V$ band $\left(\mu_{\mathrm{V}, 0}\right)$, effective surface brightness in the $V$ band $\left(\mu_{\mathrm{V}, \mathrm{e}}\right)$, Sérsic index $(n)$, and ellipticity $(\epsilon)$, divided into dwarf galaxy and GC subgroups. In general, as a group, dwarf galaxies are larger, brighter, more diffuse, less concentrated, and more elongated than GCs, but all parameters show overlap between the two classes of objects, with no clear boundaries separating the two families.

One interesting result from Figure 1 is the ellipticity distribution (bottom right panel). The vast majority of GCs are significantly round, with their ellipticities concentrated around $\epsilon<0.15$. Dwarf galaxies, on the other hand, are distributed along the whole range, preferentially at $\epsilon>0.2$, with the exception of Leo II, which shows little elongation. However, some GCs extend the distribution to significantly higher ellipticity values, overlapping with most of the dwarf galaxy distribution. In Figure 2, we show how the ellipticity behaves as a function of luminosity for all objects in our catalog. Most luminous GCs, up to $M_{\mathrm{V}} \sim-6$, have ellipticities consistent with little or no elongation. This changes at lower luminosities, where GCs are characterized by progressively increasing ellipticities, up to $\sim 0.7$ for the faintest object.

\subsection{Effect of Low Numbers of Member Stars on Measured Parameters}

Martin et al. (2008) showed that rounder objects can mistakenly seem elongated if their structural parameters are 
Table 1

Outer Halo Object Parameters Used in This Work

\begin{tabular}{|c|c|c|c|c|c|c|c|}
\hline Object & Type & $M_{\mathrm{V}}$ & $\begin{array}{c}\mu_{\mathrm{V}, 0} \\
\left(\mathrm{mag} /{ }^{\prime \prime 2}\right)\end{array}$ & $\underset{\left(\mathrm{mag} /{ }^{\prime \prime 2}\right)}{\left.\mu_{\mathrm{V}}\right)}$ & $\begin{array}{c}R_{\mathrm{e}} \\
(\mathrm{pc})\end{array}$ & $n$ & $\epsilon$ \\
\hline$\overline{\mathrm{AM}} 1$ & Outer Halo GC & $-5.02 \pm 0.26$ & $23.19_{-0.40}^{+0.39}$ & $25.18_{-0.40}^{+0.39}$ & $16.50 \pm 1.08$ & $1.08 \pm 0.13$ & $0.16 \pm 0.06$ \\
\hline $\mathrm{AM} 4$ & Outer Halo GC & $-0.89 \pm 0.81$ & $24.74_{-1.25}^{+1.18}$ & $27.51_{-1.25}^{+1.18}$ & $7.34 \pm 1.35$ & $1.44 \pm 0.33$ & $0.29 \pm 0.14$ \\
\hline Balbinot 1 & Outer Halo GC & $-1.21 \pm 0.89$ & $24.38_{-1.20}^{+1.16}$ & $27.24_{-1.20}^{+1.16}$ & $7.79 \pm 1.02$ & $1.48 \pm 0.23$ & $0.35 \pm 0.10$ \\
\hline Bootes I & UFD & $-6.00 \pm 0.25$ & $28.40 \pm 0.31$ & $29.43 \pm 0.31$ & $216.18 \pm 5.18$ & $0.64 \pm 0.03$ & $0.25 \pm 0.02$ \\
\hline Bootes II & UFD & $-2.92 \pm 0.74$ & $27.56_{-1.08}^{+1.04}$ & $28.75_{-1.08}^{+1.04}$ & $37.26 \pm 5.50$ & $0.71 \pm 0.43$ & $0.24 \pm 0.12$ \\
\hline CVn I & UFD & $-8.48 \pm 0.13$ & $27.10 \pm 0.19$ & $28.44 \pm 0.19$ & $486.38 \pm 14.59$ & $0.78 \pm 0.04$ & $0.46 \pm 0.02$ \\
\hline CVn II & UFD & $-4.85 \pm 0.36$ & $26.83_{-0.72}^{+0.67}$ & $27.76_{-0.72}^{+0.67}$ & $70.28 \pm 10.70$ & $0.59 \pm 0.49$ & $0.46 \pm 0.11$ \\
\hline Carina & $\mathrm{dSph}$ & $-9.42 \pm 0.05$ & $25.27 \pm 0.07$ & $26.74 \pm 0.07$ & $312.76 \pm 3.36$ & $0.84 \pm 0.02$ & $0.37 \pm 0.01$ \\
\hline ComBer & UFD & $-4.36 \pm 0.25$ & $26.99_{-0.37}^{+0.36}$ & $28.66_{-0.37}^{+0.36}$ & $72.06 \pm 3.84$ & $0.93 \pm 0.12$ & $0.37 \pm 0.05$ \\
\hline Draco & $\mathrm{dSph}$ & $-8.70 \pm 0.05$ & $25.01 \pm 0.07$ & $26.74 \pm 0.07$ & $207.15 \pm 1.99$ & $0.96 \pm 0.02$ & $0.30 \pm 0.01$ \\
\hline Eridanus & Outer Halo GC & $-4.92 \pm 0.26$ & $23.24 \pm 0.40$ & $25.45 \pm 0.40$ & $16.77 \pm 1.05$ & $1.18 \pm 0.14$ & $0.09 \pm 0.04$ \\
\hline Eridanus II & UFD & $-7.19 \pm 0.09$ & $26.64_{-0.31}^{+0.29}$ & $27.96_{-0.31}^{+0.29}$ & $200.07 \pm 18.79$ & $0.77 \pm 0.19$ & $0.37 \pm 0.06$ \\
\hline Eridanus III & Not classified & $-7.19 \pm 0.09$ & $18.01_{-3.51}^{+1.36}$ & $21.22_{-3.51}^{+1.36}$ & $7.34 \pm 5.82$ & $1.64 \pm 0.27$ & $0.32 \pm 0.13$ \\
\hline Fornax & $\mathrm{dSph}$ & $-13.45 \pm 0.14$ & $23.60_{-0.17}^{+0.16}$ & $24.79_{-0.17}^{+0.16}$ & $786.80 \pm 8.55$ & $0.71 \pm 0.01$ & $0.28 \pm 0.01$ \\
\hline Grus 1 & UFD & $-3.46 \pm 0.59$ & $26.87_{-1.76}^{+1.35}$ & $29.41_{-1.76}^{+1.35}$ & $72.61 \pm 30.37$ & $1.33 \pm 0.31$ & $0.54 \pm 0.26$ \\
\hline Hercules & UFD & $-5.19 \pm 0.45$ & $27.47_{-0.67}^{+0.65}$ & $29.70_{-0.67}^{+0.65}$ & $230.00 \pm 22.27$ & $1.19 \pm 0.17$ & $0.69 \pm 0.04$ \\
\hline Horologium I & UFD & $-3.53 \pm 0.56$ & $26.29_{-1.10}^{+0.99}$ & $28.07_{-1.10}^{+0.99}$ & $35.39 \pm 7.81$ & $0.98 \pm 0.47$ & $0.31 \pm 0.16$ \\
\hline Horologium II & UFD & $-1.54 \pm 1.02$ & $27.66_{-2.37}^{+1.85}$ & $29.67_{-2.37}^{+1.85}$ & $64.21 \pm 29.72$ & $1.09 \pm 0.37$ & $0.86 \pm 0.19$ \\
\hline Hydra II & UFD & $-4.58 \pm 0.37$ & $26.15_{-0.89}^{+0.79}$ & $28.40_{-0.89}^{+0.79}$ & $58.47 \pm 12.47$ & $1.20 \pm 0.46$ & $0.17 \pm 0.13$ \\
\hline Indus 1 & Not classified & $-3.31 \pm 0.62$ & $24.39_{-2.20}^{+1.53}$ & $26.69_{-2.20}^{+1.53}$ & $25.31 \pm 13.09$ & $1.22 \pm 0.44$ & $0.72 \pm 0.29$ \\
\hline Koposov 1 & Outer Halo GC & $-1.03 \pm 0.69$ & $25.11_{-1.32}^{+1.18}$ & $27.51_{-1.32}^{+1.18}$ & $10.12 \pm 2.53$ & $1.27 \pm 0.56$ & $0.55 \pm 0.15$ \\
\hline Koposov 2 & Outer Halo GC & $-0.91 \pm 0.81$ & $23.40_{-1.32}^{+1.22}$ & $25.98_{-1.32}^{+1.22}$ & $4.34 \pm 0.91$ & $1.35 \pm 0.70$ & $0.48 \pm 0.12$ \\
\hline Laevens 1 & Outer Halo GC & $-4.79 \pm 0.33$ & $24.50_{-0.67}^{+0.62}$ & $25.81_{-0.67}^{+0.62}$ & $20.67 \pm 2.95$ & $0.77 \pm 0.36$ & $0.11 \pm 0.10$ \\
\hline Laevens 2 & UFD & $-1.59 \pm 0.76$ & $25.74_{-1.24}^{+1.15}$ & $28.53_{-1.24}^{+1.15}$ & $17.45 \pm 3.49$ & $1.45 \pm 0.45$ & $0.39 \pm 0.11$ \\
\hline Leo I & $\mathrm{dSph}$ & $-11.76 \pm 0.28$ & $22.62 \pm 0.30$ & $23.93 \pm 0.30$ & $243.82 \pm 2.22$ & $0.77 \pm 0.02$ & $0.30 \pm 0.01$ \\
\hline Leo II & $\mathrm{dSph}$ & $-9.73 \pm 0.04$ & $24.25 \pm 0.06$ & $25.43 \pm 0.06$ & $168.09 \pm 2.03$ & $0.71 \pm 0.02$ & $0.07 \pm 0.02$ \\
\hline Leo IV & UFD & $-4.98 \pm 0.26$ & $27.82_{-0.54}^{+0.51}$ & $29.33_{-0.54}^{+0.51}$ & $116.92 \pm 13.89$ & $0.86 \pm 0.26$ & $0.19 \pm 0.09$ \\
\hline Leo $\mathrm{T}$ & UFD & $-7.59 \pm 0.14$ & $25.43_{-0.40}^{+0.37}$ & $27.31_{-0.40}^{+0.37}$ & $151.63 \pm 16.98$ & $1.03 \pm 0.26$ & $0.23 \pm 0.09$ \\
\hline Leo $\mathrm{V}$ & UFD & $-4.39 \pm 0.36$ & $24.90_{-0.90}^{+0.79}$ & $28.24_{-0.90}^{+0.79}$ & $51.78 \pm 11.39$ & $1.70 \pm 0.36$ & $0.35 \pm 0.07$ \\
\hline Muñoz 1 & Outer Halo GC & $-0.48 \pm 0.97$ & $26.34_{-1.42}^{+1.34}$ & $30.08_{-1.42}^{+1.34}$ & $22.25 \pm 4.19$ & $1.89 \pm 0.31$ & $0.50 \pm 0.05$ \\
\hline NGC 2419 & Outer Halo GC & $-9.33 \pm 0.03$ & $18.83 \pm 0.05$ & $22.19 \pm 0.05$ & $25.71 \pm 0.24$ & $1.71 \pm 0.02$ & $0.05 \pm 0.01$ \\
\hline NGC 5694 & Outer Halo GC & $-7.93 \pm 0.09$ & $13.42 \pm 0.14$ & $20.01 \pm 0.14$ & $4.28 \pm 0.10$ & $3.20 \pm 0.08$ & $0.06 \pm 0.02$ \\
\hline NGC 5824 & Outer Halo GC & $-9.28 \pm 0.04$ & $11.15 \pm 0.08$ & $19.09 \pm 0.08$ & $4.95 \pm 0.09$ & $3.82 \pm 0.05$ & $0.04 \pm 0.01$ \\
\hline NGC 6229 & Outer Halo GC & $-8.03 \pm 0.16$ & $13.88 \pm 0.22$ & $19.21 \pm 0.22$ & $3.19 \pm 0.09$ & $2.62 \pm 0.08$ & $0.02 \pm 0.01$ \\
\hline NGC 7006 & Outer Halo GC & $-7.41 \pm 0.08$ & $15.99 \pm 0.13$ & $21.17 \pm 0.13$ & $6.11 \pm 0.12$ & $2.55 \pm 0.07$ & $0.07 \pm 0.01$ \\
\hline NGC 7492 & Outer Halo GC & $-6.10 \pm 0.04$ & $21.24 \pm 0.06$ & $23.05 \pm 0.06$ & $9.56 \pm 0.08$ & $1.00 \pm 0.02$ & $0.02 \pm 0.02$ \\
\hline Palomar 13 & Outer Halo GC & $-2.82 \pm 0.55$ & $22.15_{-0.71}^{+0.70}$ & $26.61_{-0.71}^{+0.70}$ & $9.53 \pm 0.68$ & $2.22 \pm 0.19$ & $0.10 \pm 0.06$ \\
\hline Palomar 14 & Outer Halo GC & $-5.39 \pm 0.24$ & $23.59 \pm 0.33$ & $26.47 \pm 0.33$ & $32.04 \pm 1.34$ & $1.49 \pm 0.08$ & $0.11 \pm 0.04$ \\
\hline Palomar 15 & Outer Halo GC & $-5.65 \pm 0.19$ & $23.07 \pm 0.24$ & $24.97 \pm 0.24$ & $19.02 \pm 0.39$ & $1.04 \pm 0.06$ & $0.05 \pm 0.02$ \\
\hline Palomar 2 & Outer Halo GC & $-9.05 \pm 0.07$ & $16.57_{-0.12}^{+0.11}$ & $19.88_{-0.12}^{+0.11}$ & $7.83 \pm 0.16$ & $1.69 \pm 0.04$ & $0.05 \pm 0.02$ \\
\hline Palomar 3 & Outer Halo GC & $-5.48 \pm 0.21$ & $23.55_{-0.28}^{+0.27}$ & $25.08_{-0.28}^{+0.27}$ & $19.37 \pm 0.54$ & $0.87 \pm 0.05$ & $0.07 \pm 0.03$ \\
\hline Palomar 4 & Outer Halo GC & $-6.01 \pm 0.16$ & $22.74_{-0.23}^{+0.22}$ & $24.81_{-0.23}^{+0.22}$ & $20.24 \pm 0.63$ & $1.12 \pm 0.08$ & $0.03 \pm 0.02$ \\
\hline Phoenix 2 & Not classified & $-3.28 \pm 0.63$ & $25.85_{-1.03}^{+0.97}$ & $27.97_{-1.03}^{+0.97}$ & $38.87 \pm 6.52$ & $1.14 \pm 0.27$ & $0.61 \pm 0.15$ \\
\hline Pictoris 1 & Not classified & $-3.44 \pm 0.60$ & $24.51_{-2.04}^{+1.46}$ & $27.43_{-2.04}^{+1.46}$ & $21.89 \pm 10.61$ & $1.51 \pm 0.31$ & $0.24 \pm 0.19$ \\
\hline Pisces II & UFD & $-4.21 \pm 0.38$ & $26.53_{-0.77}^{+0.71}$ & $28.61_{-0.77}^{+0.71}$ & $64.59 \pm 10.59$ & $1.12 \pm 0.34$ & $0.40 \pm 0.10$ \\
\hline Pyxis & Outer Halo GC & $-5.69 \pm 0.19$ & $23.07_{-0.25}^{+0.24}$ & $24.87_{-0.25}^{+0.24}$ & $18.57 \pm 0.46$ & $0.99 \pm 0.05$ & $0.04 \pm 0.02$ \\
\hline Reticulum II & UFD & $-3.86 \pm 0.38$ & $26.79 \pm 0.46$ & $27.73 \pm 0.46$ & $48.78 \pm 1.83$ & $0.60 \pm 0.05$ & $0.56 \pm 0.03$ \\
\hline Sculptor & $\mathrm{dSph}$ & $-10.81 \pm 0.14$ & $23.41_{-0.38}^{+0.36}$ & $24.66_{-0.38}^{+0.36}$ & $215.14 \pm 22.51$ & $0.74 \pm 0.07$ & $0.26 \pm 0.01$ \\
\hline Segue 1 & UFD & $-1.29 \pm 0.73$ & $28.08_{-1.01}^{+0.98}$ & $29.57_{-1.01}^{+0.98}$ & $26.43 \pm 3.21$ & $0.85 \pm 0.28$ & $0.34 \pm 0.11$ \\
\hline Segue 2 & UFD & $-1.85 \pm 0.88$ & $28.49_{-1.06}^{+1.05}$ & $29.92_{-1.06}^{+1.05}$ & $37.06 \pm 2.95$ & $0.82 \pm 0.16$ & $0.21 \pm 0.07$ \\
\hline Segue 3 & Outer Halo GC & $-0.85 \pm 0.67$ & $23.86_{-1.08}^{+1.02}$ & $26.32_{-1.08}^{+1.02}$ & $4.08 \pm 0.71$ & $1.30 \pm 0.30$ & $0.22 \pm 0.09$ \\
\hline Sextans & $\mathrm{dSph}$ & $-8.71 \pm 0.06$ & $27.23 \pm 0.08$ & $28.18 \pm 0.08$ & $442.04 \pm 4.25$ & $0.60 \pm 0.01$ & $0.30 \pm 0.01$ \\
\hline UMa I & UFD & $-5.12 \pm 0.38$ & $29.12_{-0.48}^{+0.47}$ & $29.78_{-0.48}^{+0.47}$ & $235.32 \pm 9.59$ & $0.47 \pm 0.08$ & $0.57 \pm 0.03$ \\
\hline UMa II & UFD & $-4.23 \pm 0.26$ & $28.08 \pm 0.33$ & $29.66 \pm 0.33$ & $129.85 \pm 4.28$ & $0.89 \pm 0.10$ & $0.56 \pm 0.03$ \\
\hline UMi & $\mathrm{dSph}$ & $-9.02 \pm 0.05$ & $25.77 \pm 0.06$ & $27.09 \pm 0.06$ & $367.21 \pm 2.43$ & $0.77 \pm 0.01$ & $0.55 \pm 0.01$ \\
\hline Whiting 1 & Outer Halo GC & $-2.54 \pm 0.44$ & $21.45_{-0.66}^{+0.64}$ & $25.84_{-0.66}^{+0.64}$ & $6.39 \pm 0.61$ & $2.19 \pm 0.26$ & $0.24 \pm 0.05$ \\
\hline Willman I & UFD & $-2.52 \pm 0.74$ & $25.88_{-0.94}^{+0.92}$ & $28.43_{-0.94}^{+0.92}$ & $27.97 \pm 2.43$ & $1.34 \pm 0.20$ & $0.47 \pm 0.06$ \\
\hline
\end{tabular}



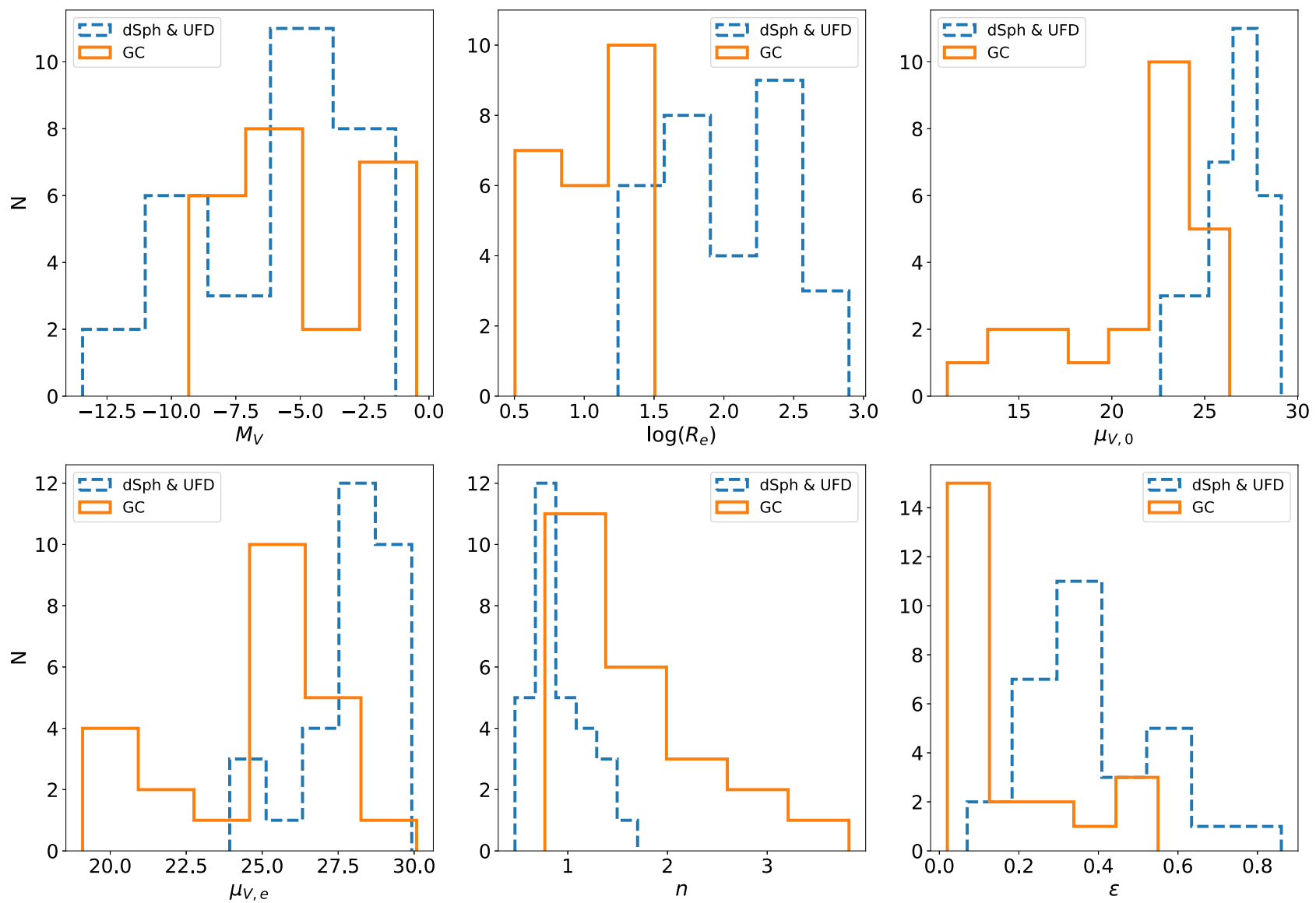

Figure 1. Comparison of the GC and dwarf dSph parameter distributions, for all structural and photometric parameters analyzed in our study.

measured from samples with low numbers of stars. Similar studies have shown that the low number of stars detected in ultra-low-luminosity objects affect our ability to reliably measure their structural properties (e.g., Sand et al. 2010; Muñoz et al. 2012b).

To explore the effect of low numbers of member stars on the measured ellipticities, we fit a stellar density profile over a set of simulated stellar overdensities of different numbers of member stars. To simulate a stellar overdensity, we generate member stars randomly across a defined field of view using a Sérsic density profile as a probability distribution. Given that we want to test the potential departure from a round shape at low luminosities, we set the ellipticity of our simulated object equal to 0 . We run two sets of simulations, where the effective radius is kept constant at $1 ! 25$, and we give two different values to the Sérsic index, 1 and 4 , in order to see the effect of different concentrations. The field of view is given by a 30 arcmin $\times 30$ arcmin area, and, for simplicity, we put the simulated object at the center. Finally, we add a particle background given by a constant stellar background density through the entire field of view. The value we adopt for this parameter is $0.5\left[1 / \operatorname{arcmin}^{2}\right]$, which is the typical value derived by Muñoz et al. (2018b) for the objects in our data set (see their Figures 4-14, right panels).

We generate samples of different numbers of member stars by taking random subsamples without replacement from the originally simulated object. The number of member stars for the subsamples ranges from 1 to 2000 stars in different steps given by a logarithmic scale. For every simulated subsample, we then fit a Sérsic profile with the effective radius, Sérsic index, central coordinate, and ellipticity as free parameters, while the background density is kept fixed. The fit is performed through a Bayesian MCMC approach, using the emcee Python package (Foreman-Mackey et al. 2013). The likelihood function is represented by the Sérsic density profile, and the priors for all free parameters are defined to be uniform. The density profile used is given by

$$
\Sigma(r)=\Sigma_{0, \mathrm{~S}} \exp \left[-b_{n}\left(\frac{r}{r_{e}}\right)^{1 / n}\right]+\Sigma_{\mathrm{bkg}},
$$

where $\Sigma(r)$ is the stellar density for any given radius $r, \Sigma_{0, \mathrm{~S}}$ is the Sérsic central stellar density, $n$ is the Sérsic index, $r_{e}$ is the effective radius, $b_{n}$ is approximated by $1.999 n-0.327$ (Capaccioli 1989), and $\Sigma_{\mathrm{bkg}}$ is the background stellar density.

The top panels of Figure 3 show the estimation of the ellipticity for every simulated subsample. It is evident that a low number of member stars increases the bias and the uncertainty of the estimation. This is consistent with the interpretation that the trend in ellipticity that we see in Figure 2 is possibly due to the low luminosity of the satellite objects and not a real effect.

In the same vein, it is likely that the ellipticity measurements of dSphs at low luminosities are also affected by low numbers of member stars. Therefore, for absolute magnitudes fainter than $\sim-5$, we cannot reliably use the ellipticity values, and thus we cannot clearly establish differences or similarities in ellipticities for GCs and dSphs.

In Figure 3, we also show the behavior of the Sérsic index, effective radius, and central surface brightness as a function of 


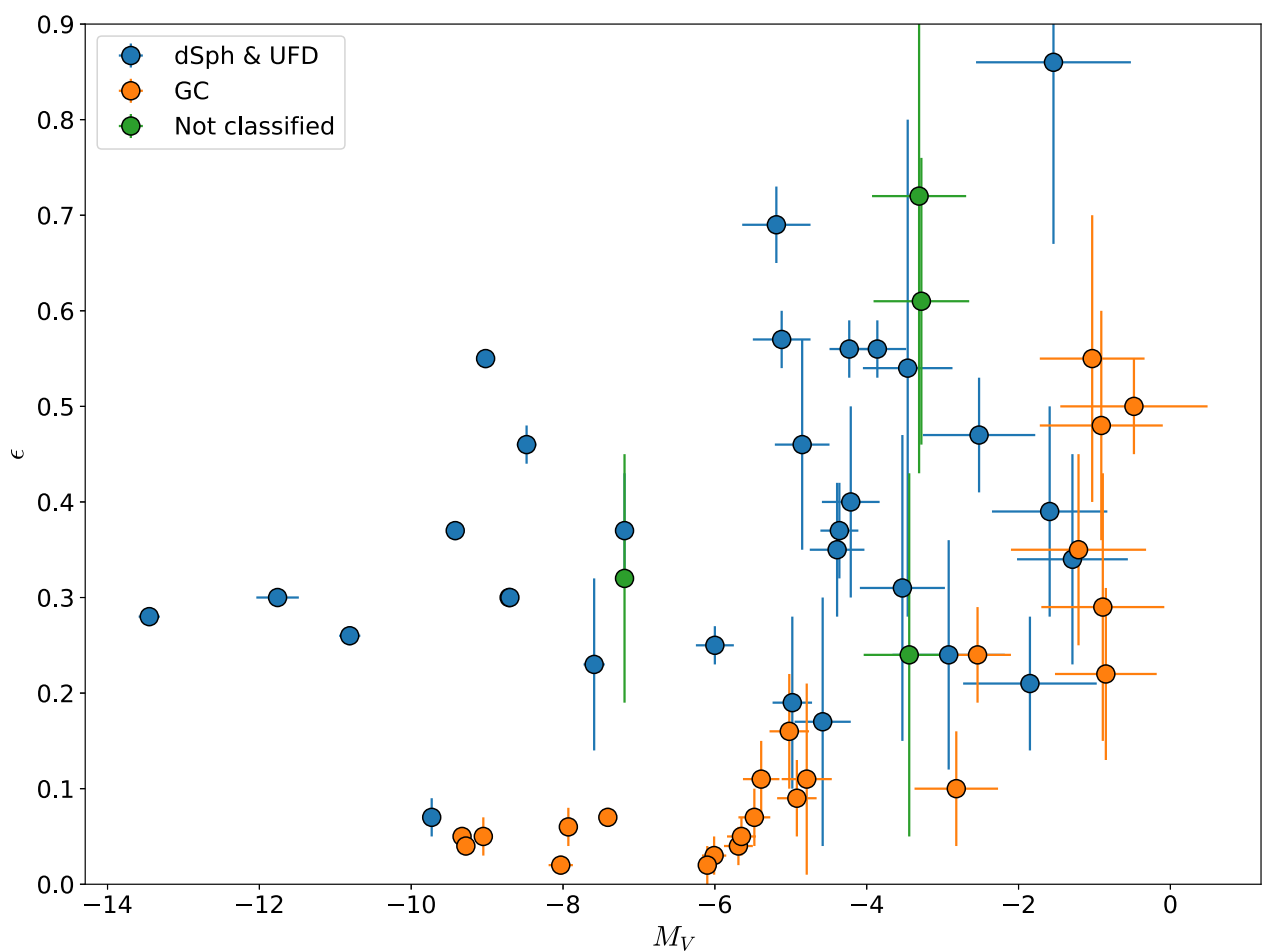

Figure 2. Evolution of ellipticity with luminosity for GCs, dSphs, and UFDs. GCs with luminosities higher than $M_{\mathrm{V}} \sim-5$ are clearly more round than dwarf galaxies of comparable luminosity. At lower luminosities, clusters seem to increase their ellipticities to values similar to those of galaxies.

the number of stars. All of these parameters appear more robust to the shot noise introduced by the low number of stars, with significant deviations observed only in the more extreme cases of fewer than $\sim 30$ stars for the $n=1$ case, and fewer than $\sim 100$ stars for $n=4$. Although the measured parameters for objects with true Sérsic index equal to 4 are more sensitive to the number of member stars, we note that all our objects with a high Sérsic index are dominated by a large number of member stars. We therefore regard trends involving $R_{\mathrm{e}}, n$, and $\mu_{\mathrm{V}, 0}$ as more reliable, considering that the object with the lowest number of member stars in our sample has on the order of 100 stars.

It is important to mention that the values for $\mu_{\mathrm{V}, 0}$ were not calculated directly from the simulation, but derived as in Muñoz et al. (2018b). The relations used are

$$
\begin{gathered}
I_{0}=L b_{n}^{2 n} /\left[2 \pi R_{\mathrm{e}}^{2} n \Gamma(2 n)(1-\epsilon)\right], \\
\mu_{0}=M_{\odot}+21.572-2.5 \log I_{0},
\end{gathered}
$$

where $b_{n}=1.9992 n-0.3271, I_{0}$ is the central intensity, and $L$ is the total luminosity of the object. Uncertainties were calculated by propagation of errors.

\section{4. $n-R_{\mathrm{e}}$ Correlation}

An intriguing result regarding the overall properties of GCs and dwarf galaxies is a relation between the Sérsic index and effective radii that is followed by all outer halo objects in our sample. In Figure 4, we present the relation between the Sérsic index and the effective radius for dwarf galaxies and GCs. The figure shows that the Sérsic index decreases linearly with increasing size in log space. This means that smaller satellite objects are more centrally concentrated than larger ones, because the Sersic index is a proxy for central concentration (Trujillo et al. 2001). Spearman's correlation coefficient is
-0.728 , with a $p$-value less than 0.001 , indicating that the correlation is significant at a high level. A similar trend, but in the opposite sense, has already been reported for dwarf and elliptical galaxies (Caon et al. 1993), where larger galaxies have a higher Sérsic index (i.e., are more concentrated).

The fact that both GCs and dwarf galaxies share the same locus, forming a continuous group, suggests a remarkable similarity between these two types of objects that is at first glance surprising, given that GCs and dwarf galaxies do not follow continuous trends in other structural parameter planes.

We note that, even though our structural parameters come from fitting a Sérsic profile to the number density profiles, Trujillo et al. (2001) demonstrated that a relation between $R_{\mathrm{e}}$ and $n$ cannot be produced by parameter coupling due to model fitting.

\section{Discussion}

\subsection{Origin of the $n-R_{\mathrm{e}}$ Relation}

The observed $n-R_{\mathrm{e}}$ trend for GCs and dwarf galaxies does not have an obvious interpretation, especially if one takes into account that these objects have long been considered to be intrinsically different; GCs are believed to be DM-free while dwarf galaxies have been found to be heavily DM-dominated.

Graham (2011) showed that it is possible to understand the existence of a relationship between the effective radius and mean effective surface brightness for elliptical and dwarf elliptical galaxies by showing that it can naturally arise if $\mu_{0}-M_{\mathrm{V}}$ and $\log (n)-M_{V}$ behave linearly, when both types of galaxies follow a Sérsic density profile. In what follows, we consider a similar approach to understand the $n-R_{\mathrm{e}}$ trend and show an analytic procedure to reproduce the $n-R_{\mathrm{e}}$ relation by considering linear fittings to the $\mu_{0}-M_{\mathrm{V}}$ and $n-M_{\mathrm{V}}$ plots. 

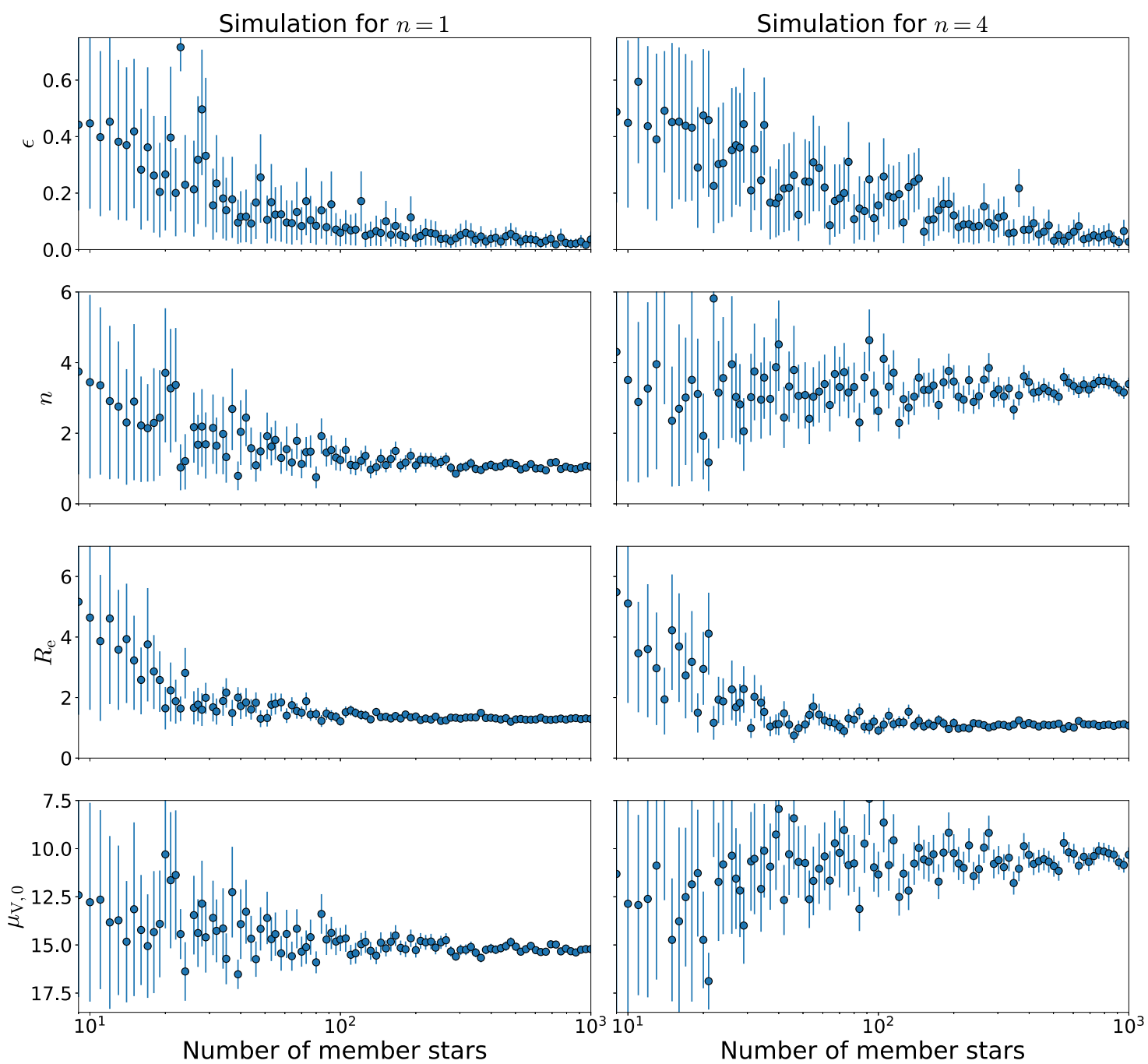

Figure 3. Ellipticity, Sérsic index, effective radius, and central surface brightness estimations for different subsamples of member stars from the simulated satellite object. The left panels are the results for $n=1$, while the right panels are for $n=4$. This plot shows that the increase in ellipticities observed in globular clusters in Figure 2 is likely due to a statistical effect of the low number of observed member stars. This effect is only seen at a much lower number of member stars for the rest of the parameters.

Note that in Graham (2011), they aimed to explain a correlation in a different parameter space than $n-R_{\mathrm{e}}$ (the one we present in this paper). However, the analytical procedure is the same. Unlike the case of elliptical galaxies, we know beforehand that GCs and dwarf galaxies do not form a single relation in either $\mu_{0}-M_{\mathrm{V}}$ and $n-M_{\mathrm{V}}$ spaces, and thus we follow Graham's (2011) procedure to investigate how the different behaviors in these parameter spaces can still result in the $n-R_{\mathrm{e}}$ trend we detected. Additionally, we use the form $n-M_{\mathrm{V}}$ instead of a $\log (n)-M_{V}$ relation. We do this because our range of $n$ is small enough that transforming to log space would not produce any substantial improvement. Moreover, by using the $n-M_{\mathrm{V}}$ relation, we avoid introducing an extra $\log (n)$ term in Equation (6).

The intensity profile at any given radius $r$ is modeled by the Sérsic profile as

$$
I(r)=I_{e} \exp \left\{-b_{n}\left[\left(\frac{r}{r_{e}}\right)^{1 / n}-1\right]\right\},
$$

where $I_{e}$ is the intensity at the effective radius $r_{e}, n$ is the Sérsic index, and $b_{n}$ is a function that depends on $n$. As demonstrated by Graham \& Driver (2005), from a Sérsic profile, it is possible to derive the following expression:

$$
M_{\mathrm{tot}}=\mu_{\mathrm{e}}-2.5 \log [f(n)]-2.5 \log \left(2 \pi R_{\mathrm{e}, \mathrm{kpc}}^{2}\right)-36.57,
$$

where $M_{\text {tot }}$ is the total absolute magnitude, $\mu_{\mathrm{e}}$ is the effective surface brightness, $R_{\mathrm{e}, \mathrm{kpc}}$ is the effective radius in kiloparsecs, $n$ is the Sérsic index, and

$$
f(n)=\frac{n \mathrm{e}^{b}}{b^{2 n}} \Gamma(2 n)
$$

with $b=1.9992 n-0.3271$ for $0.5<n<10$ (Capaccioli $1989)$, and $\Gamma$ is the gamma function.

Finally, if we consider relationships of the form

$$
\begin{gathered}
\mu_{0}=A M_{\mathrm{tot}}+B, \\
n=C M_{\mathrm{tot}}+D,
\end{gathered}
$$

where $\mu_{0}$ is the central surface brightness and the fact that $\mu_{\mathrm{e}}=$ $\mu_{0}+1.086 b$, one obtains an equation that relates the Sérsic index with the effective radius, of the form

$$
\log \left(R_{\mathrm{e}, \mathrm{kpc}}\right)=E n+F \log [f(n)]+G,
$$




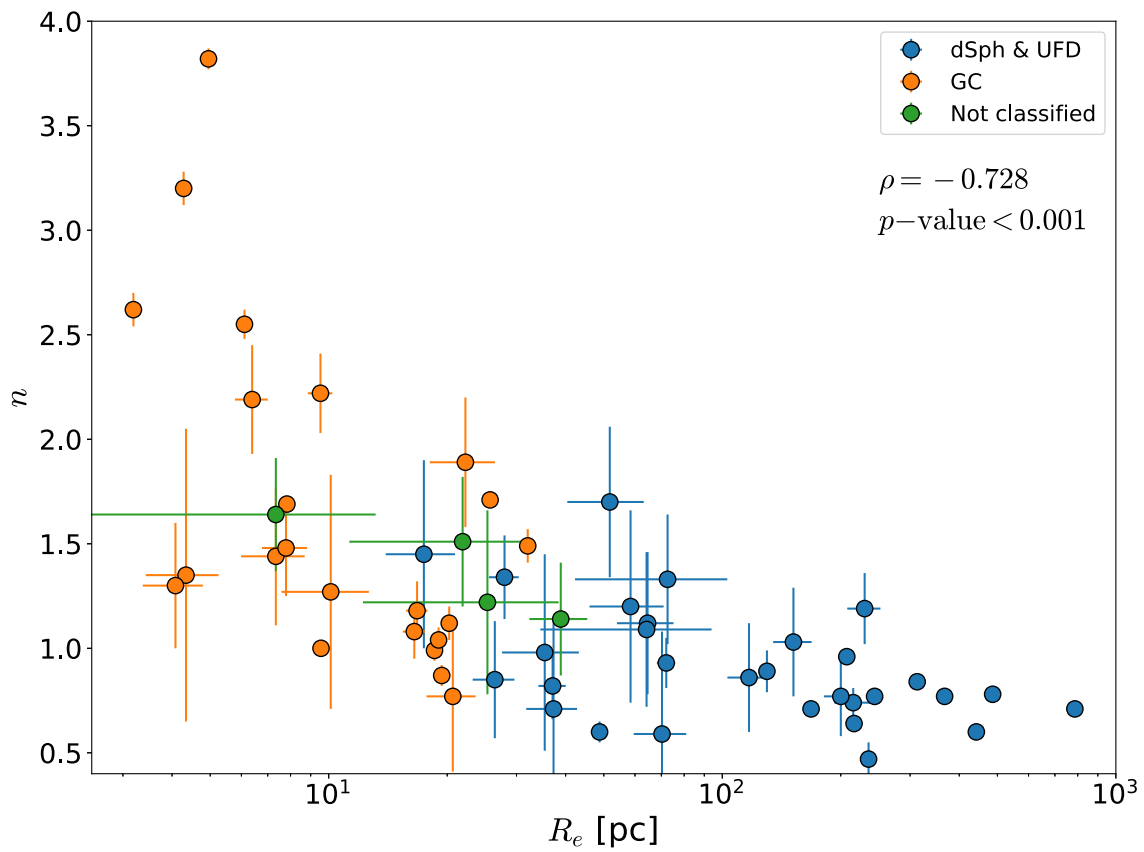

Figure 4. Correlation between the Sérsic index and the effective radius in parsecs for all the objects in our sample.
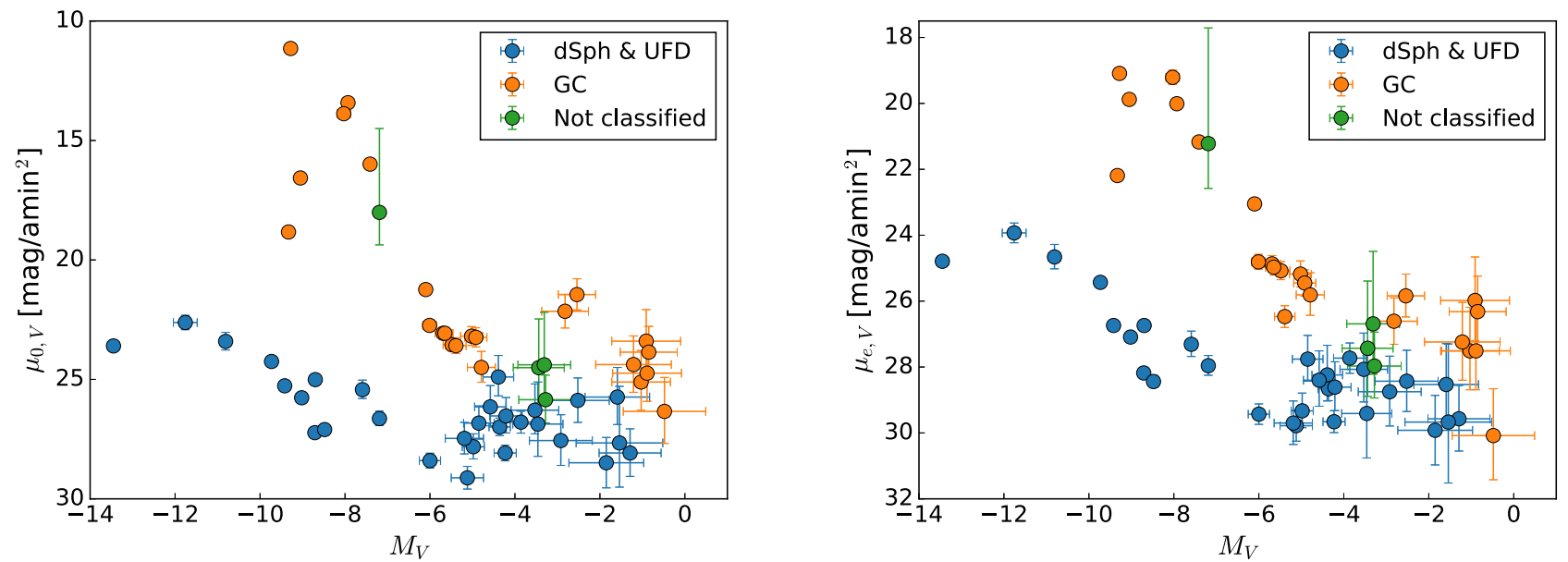

Figure 5. Comparison of surface brightness with absolute magnitude for the objects in our data set. Left panel: central surface brightness vs. absolute magnitude. Right panel: effective surface brightness vs. absolute magnitude. Note that in both panels, it is evident that dwarf galaxies and globular clusters are well separated at high luminosities $\left(M_{\mathrm{V}}<\sim 5\right)$. At lower luminosities, both groups tend to mix, although on average globular clusters still show higher surface brightnesses.

with $E=\frac{A-1}{5 C}+0.434, F=-0.5$, and $G=\frac{B}{5}-\frac{D(A-1)}{5 C}-$ 7.784 .

This procedure shows that, for a Sérsic density profile, linear relations in the $\mu_{0}-M_{\mathrm{V}}$ and $n-M_{\mathrm{V}}$ spaces reproduce a relation in the $n-R_{\mathrm{e}}$ space. One can reproduce other relationships between pairs of structural parameters if other linear relationships exist.

\subsection{Surface Brightness versus Absolute Magnitude}

Figure 5 shows the central and effective surface brightness versus absolute magnitude. In both plots, galaxies form a continuous group characterized by a luminosity versus surface brightness dependency that flattens at $M_{\mathrm{V}} \sim-6$, in the region dominated by UFDs. This flattening was already identified by McConnachie (2012), and it is possibly due to a detection bias, because the surface brightness of the least luminous UFDs are very near the detection limit of current surveys.
In the case of GCs, they show a higher central and effective surface brightness than galaxies at high luminosities (lower than $\left.M_{\mathrm{V}} \sim-4\right)$. At lower luminosities, GCs tend to concentrate at an almost constant surface brightness value, showing a similar behavior to the UFDs.

Although GCs and dwarf galaxies come closer at low luminosities in the $\mu_{0}-M_{\mathrm{V}}$ space, neither group mixes completely - GCs have a higher average surface brightness than UFDs. This is not easily explained as a detection bias, because the surface brightness values at which GCs are concentrated are higher than the detection limits. A possible explanation for this different surface brightness floor is the fact that UFDs are believed to be currently embedded in a DM halo. For a given luminosity (or stellar mass), an object inside a DM halo is likely more robust to tidal disintegration than a DM-free one and thus could reach lower luminosities, allowing also for lower surface brightnesses. 


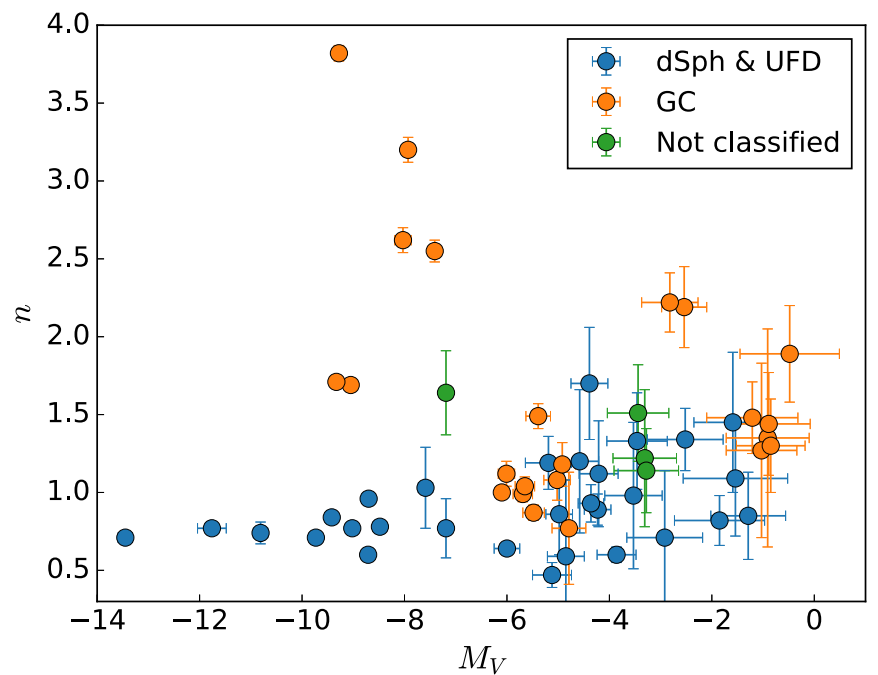

Figure 6. Sérsic index against absolute magnitude for all objects in our data set. Dwarf galaxies and globular clusters follow a nearly linear relationship that spans the whole range of luminosity, where low-luminosity objects have a slightly higher Sérsic index. There are six globular clusters located between $M_{\mathrm{V}} \sim-10$ and $M_{\mathrm{V}} \sim-8$ that do not follow this tendency, having high Sérsic indexes for their luminosities.

\subsection{Sérsic Index versus Absolute Magnitude}

Figure 6 shows the relation between the Sérsic index and the absolute magnitude. Dwarf galaxies concentrate at relatively small values of the Sérsic index, between $\sim 0.5$ and $\sim 1.5$, following a trend where the Sérsic index increases slightly at lower luminosities. On the other hand, GCs do not seem to follow a single trend. Overall, it appears that the Sérsic index increases with luminosity. However, the data allow a different interpretation: most low-luminosity GCs follow the trend delineated by dwarf galaxies, and only the six brighter clusters are off this trend and occupy a different region in the plot. In the surface brightness versus absolute magnitude relations (Figure 5), these clusters are also the ones with the highest surface brightnesses. In fact, from this Figure 5, it is also possible to infer that the low-luminosity outer halo GCs and dwarf galaxies constitute a single group (although GCs have a higher mean central and effective surface brightnesses) with the high surface brightness (HSB) GCs being outliers, which may be part of a different subgroup of clusters.

In the next subsections, we apply to our data set the procedure from which the $n-R_{\mathrm{e}}$ relation originates, in order to see if it can be reproduced by linear fits obtained from the $\mu_{0}-M_{\mathrm{V}}$ and $n-M_{\mathrm{V}}$ plots.

\subsection{Linear Fits to the $\mu_{0}-M_{\mathrm{V}}$ and $n-M_{\mathrm{V}}$ Relations}

As a first approach, we fit linear relations to dwarf galaxies and GCs assuming that they constitute two separate groups, following the conventional classification for each object. The left panel of Figure 7 shows the linear fit to the central surface brightness versus absolute magnitude relation for GCs and galaxies. The fit for galaxies is given by

$$
\mu_{\mathrm{V}, 0}=(0.569 \pm 0.120) M_{\mathrm{V}}+(30.597 \pm 1.105),
$$

while for GCs, the fit is given by

$$
\mu_{\mathrm{V}, 0}=(1.104 \pm 0.194) M_{\mathrm{V}}+(26.598 \pm 1.091) .
$$

Next, we analyze the relationship between the Sérsic index and absolute magnitude for dwarf galaxies, which is presented in the middle panel of Figure 7. The relations are characterized by

$$
n=(0.036 \pm 0.015) M_{\mathrm{V}}+(1.124 \pm 0.101),
$$

while for GCs, the fit is given by

$$
n=(-0.100 \pm 0.053) M_{\mathrm{V}}+(1.184 \pm 0.300) .
$$

Finally, we obtain two relations similar to Equation (6), one for dwarf galaxies and another for GCs. For galaxies, the relation is

$$
\begin{aligned}
& \log _{10}\left(R_{\mathrm{e}, \mathrm{kpc}}\right)=(-1.965 \pm 1.211) n \\
& \quad+-0.500 \log _{10}(f(n))+(1.033 \pm 1.401)
\end{aligned}
$$

while for GCs the relation is

$$
\begin{aligned}
& \log _{10}\left(R_{\mathrm{e}, \mathrm{kpc}}\right)=(0.226 \pm 0.406) n \\
& \quad+-0.500 \log _{10}(f(n))+(-2.218 \pm 0.531) .
\end{aligned}
$$

These derived relations are overplotted on our data in the right panel of Figure 7. Dwarf galaxies seem to follow the predicted relation, represented by a blue solid line. On the contrary, GCs do not follow their predicted relation, represented by an orange solid line. This shows that separating our data into two groups, one composed of GCs and the other of $\mathrm{dSphs}$, and fitting linear relations in the $\mu_{0}-M_{\mathrm{V}}$ and $n-M_{\mathrm{V}}$ parameter spaces do not explain completely the observed correlation observed in the $n-R_{\mathrm{e}}$ parameter space. This is expected for GCs, because it is clear that a linear fit in $n-M_{\mathrm{V}}$ for these objects is not a good model.

\subsection{Two Separate GC Populations}

It is interesting that some of the GCs seem to follow the extrapolation of the $n-R_{\mathrm{e}}$ relation for dwarf galaxies. This prompts us to revisit the idea of two different GCs groups and consider the possibility that some outer halo GCs do not constitute a different group from UFDs.

To further explore the origin of the $n-R_{\mathrm{e}}$ correlation, we add to our sample the inner GC data from Carballo-Bello et al. (2012), covering a range in galactocentric radius from 11 to $21 \mathrm{kpc}$. We estimated their Sérsic index and effective radius by fitting a Sérsic profile to radial density profiles through an MCMC fitting procedure, where the free parameters are the Sérsic index, the effective radius, and the central surface density, and we used flat priors to estimate them. Given a degeneracy when estimating the Sérsic index and the background surface density, we fixed the latter by visually exploring the density profiles for each inner cluster. We also obtained central surface brightness and absolute magnitude values from Harris (1996; 2010 edition). Additionally, we add parameters of the object Kim 1 from the DES data set, which where calculated in Muñoz et al. (2018b). It is relevant to mention that adding the inner halo GCs to the data sets breaks its homogeneity. However, this only affects the HSB group, keeping the homogeneity for the LSB clusters + galaxies group intact.

Table 2 shows the estimated parameters for inner GCs, and Figure 8 shows our Sérsic profile fit to the radial density profiles of inner halo GCs. Figure 9 shows the same plots as 

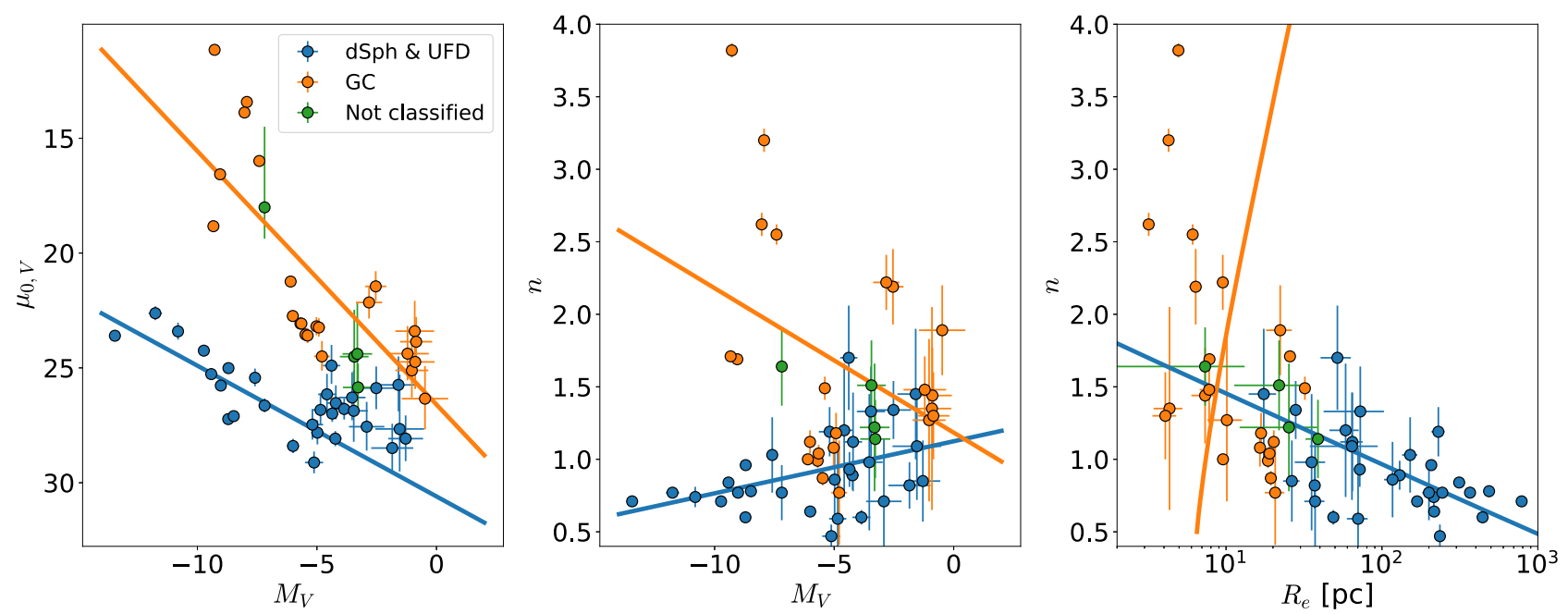

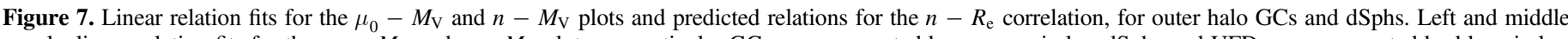

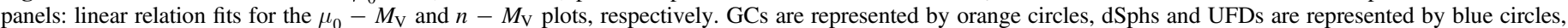

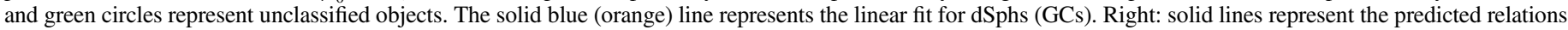
for the $n-R_{\mathrm{e}}$ correlation for GCs and dwarf galaxies. Colors and symbols follow the same convention as the previous panels.

Table 2

Parameters for Inner Halo GCs

\begin{tabular}{lrcrc}
\hline \hline Object & $M_{\mathrm{V}}$ & $\begin{array}{c}\mu_{\mathrm{V}, 0} \\
\left(\mathrm{mag} / \mathrm{arcsec}^{2}\right)\end{array}$ & $\begin{array}{c}R_{\mathrm{e}} \\
(\mathrm{pc})\end{array}$ & $n$ \\
\hline Kim 1 & 0.74 & 25.22 & $5.36 \pm 1.27$ & $1.24 \pm 0.55$ \\
NGC 1261 & -7.80 & 17.73 & $4.75 \pm 0.12$ & $1.73 \pm 0.05$ \\
NGC 1851 & -8.33 & 14.25 & $1.85 \pm 0.04$ & $3.68 \pm 0.09$ \\
NGC 1904 & -7.86 & 16.02 & $3.17 \pm 0.04$ & $2.21 \pm 0.03$ \\
NGC 2298 & -6.31 & 18.90 & $3.02 \pm 0.06$ & $1.49 \pm 0.04$ \\
NGC 4147 & -6.17 & 17.38 & $2.94 \pm 0.06$ & $2.40 \pm 0.07$ \\
NGC 4590 & -7.37 & 18.81 & $5.46 \pm 0.17$ & $1.94 \pm 0.08$ \\
NGC 5024 & -8.71 & 17.38 & $7.63 \pm 0.14$ & $2.06 \pm 0.05$ \\
NGC 5053 & -6.76 & 22.03 & $11.08 \pm 0.40$ & $1.06 \pm 0.08$ \\
NGC 5272 & -8.88 & 16.64 & $3.92 \pm 0.22$ & $3.05 \pm 0.15$ \\
NGC 5466 & -6.98 & 21.61 & $6.91 \pm 1.13$ & $2.08 \pm 0.31$ \\
NGC 5634 & -7.69 & 17.20 & $5.08 \pm 0.18$ & $2.23 \pm 0.12$ \\
NGC 6864 & -8.57 & 15.52 & $2.70 \pm 0.09$ & $2.36 \pm 0.09$ \\
NGC 7078 & -9.19 & 14.21 & $3.40 \pm 0.05$ & $2.50 \pm 0.05$ \\
Palomar 5 & -5.17 & 24.64 & $21.86 \pm 1.10$ & $1.16 \pm 0.15$ \\
Ruprecht 106 & -6.35 & 21.82 & $12.98 \pm 0.50$ & $0.64 \pm 0.07$ \\
\hline
\end{tabular}

Figure 7, but this time including the inner GCs mentioned before. As can be seen in the central surface brightness versus absolute magnitude plot (left panel), most inner GCs are located in a high-luminosity, high central surface brightness area, in comparison to most outer halo clusters. There are four inner halo clusters (purple circles) separated from the main group of inner halo clusters, below the $\mu_{\mathrm{V}, 0} \sim 20$ line. Additionally, some outer halo clusters (orange stars) are mixed with the inner halo GCs and separated from the rest of the outer halo cluster population (orange circles), which is located close to the UFD group. The separation between the two GC groups seems to be also marked by the $\mu_{\mathrm{V}, 0} \sim 20$ line. We tentatively name the groups of clusters above this line the High Surface Brightness (HSB) group, while the group of clusters below this division line is the Low Surface Brightness (LSB) group.

The middle panel of Figure 9, Sérsic index versus absolute magnitude, also shows the separation of the HSB and LSB groups. Although the HSB group is distributed between $-10<M_{\mathrm{V}}<-5$ and $1<n<4$, the LSB group occupies the region $-4<M_{\mathrm{V}}$ and $n \lesssim 2$ and mix with the lowluminosity part of the dwarf galaxy group.

Finally, in the $n-R_{\mathrm{e}}$ plot, right panel in Figure 9, the HSB clusters are concentrated in the upper left region of the plot and deviate from the main correlation composed of LSB clusters and dwarf galaxies.

Adding the inner halo clusters to our outer halo sample reinforces the notion that there might be two subgroups of satellite objects: one composed of high-luminosity, high central surface brightness clusters from the MW's inner and outer halos, and another composed of dwarf galaxies and GCs of lower central surface brightness and in general lower luminosity, which share the same parameter space occupied by low-luminosity galaxies. With this in mind, we explore if fitting different linear relations to both groups defined above will reproduce the distribution in the Sérsic index versus effective radius plot.

To find the relation in the $n-R_{\mathrm{e}}$ parameter space for the LSB GC + dwarf galaxy and HSB GC groups, we repeat the same procedure as before. For the LSB GCs + dwarf galaxies, the equations of empirical linear fit are

$$
\begin{gathered}
\mu_{\mathrm{V}, 0}=(0.141 \pm 0.091) M_{\mathrm{V}}+(25.811 \pm 0.524), \\
n=(0.062 \pm 0.016) M_{\mathrm{V}}+(1.396 \pm 0.092),
\end{gathered}
$$

and its $n-R_{\mathrm{e}}$ relation is

$$
\begin{aligned}
& \log _{10}\left(R_{\mathrm{e}, \mathrm{kpc}}\right)=(-2.356 \pm 0.783) n \\
& \quad+-0.500 \log _{10}(f(n))+(1.273 \pm 1.128) .
\end{aligned}
$$

For the HSB GC group, the empirical linear fit equations are

$$
\begin{aligned}
& \mu_{\mathrm{V}, 0}=(4.190 \pm 1.985) M_{\mathrm{V}}+(50.439 \pm 25.170), \\
& n=(-2.261 \pm 1.675) M_{\mathrm{V}}+(-15.848 \pm 11.878),
\end{aligned}
$$

and its $n-R_{\mathrm{e}}$ relation is

$$
\begin{aligned}
& \log _{10}\left(R_{\mathrm{e}, \mathrm{kpc}}\right)=(0.152 \pm 0.273) n \\
& \quad+-0.500 \log _{10}(f(n))+(-2.168 \pm 7.435) .
\end{aligned}
$$

To fit the $\mu_{0}-M_{\mathrm{V}}$ and $n-M_{\mathrm{V}}$ relations for HSB clusters, we in practice consider the $\mu_{\mathrm{V}, 0}$ and $n$ parameters as 

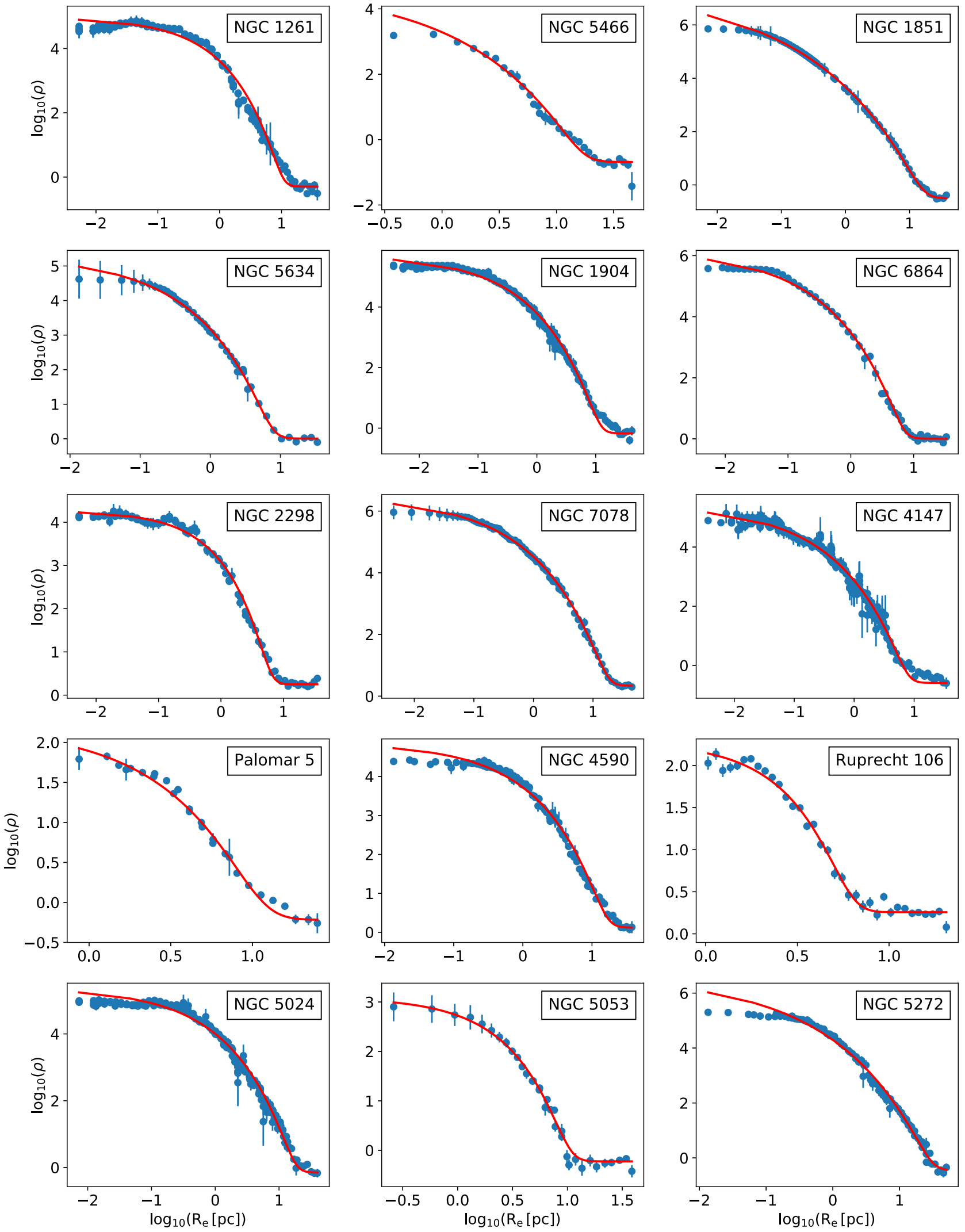

Figure 8. Radial density profiles (blue circles) and fitted Sérsic profile (red line) for each of the inner halo GCs from Carballo-Bello et al. (2012).

independent variables and $M_{\mathrm{V}}$ as the dependent variable, because the distribution of clusters on those parameter spaces is strongly vertical when assuming $M_{\mathrm{V}}$ as the independent variable. Then, we invert the equations to obtain the coefficients when $M_{\mathrm{V}}$ acts as the independent variable. The results of this last procedure are shown in Figure 9. The left and middle panels show the linear fits to the $\mu_{0}-M_{\mathrm{V}}$ and $n-M_{\mathrm{V}}$ plots, respectively. In each parameter space, the red 

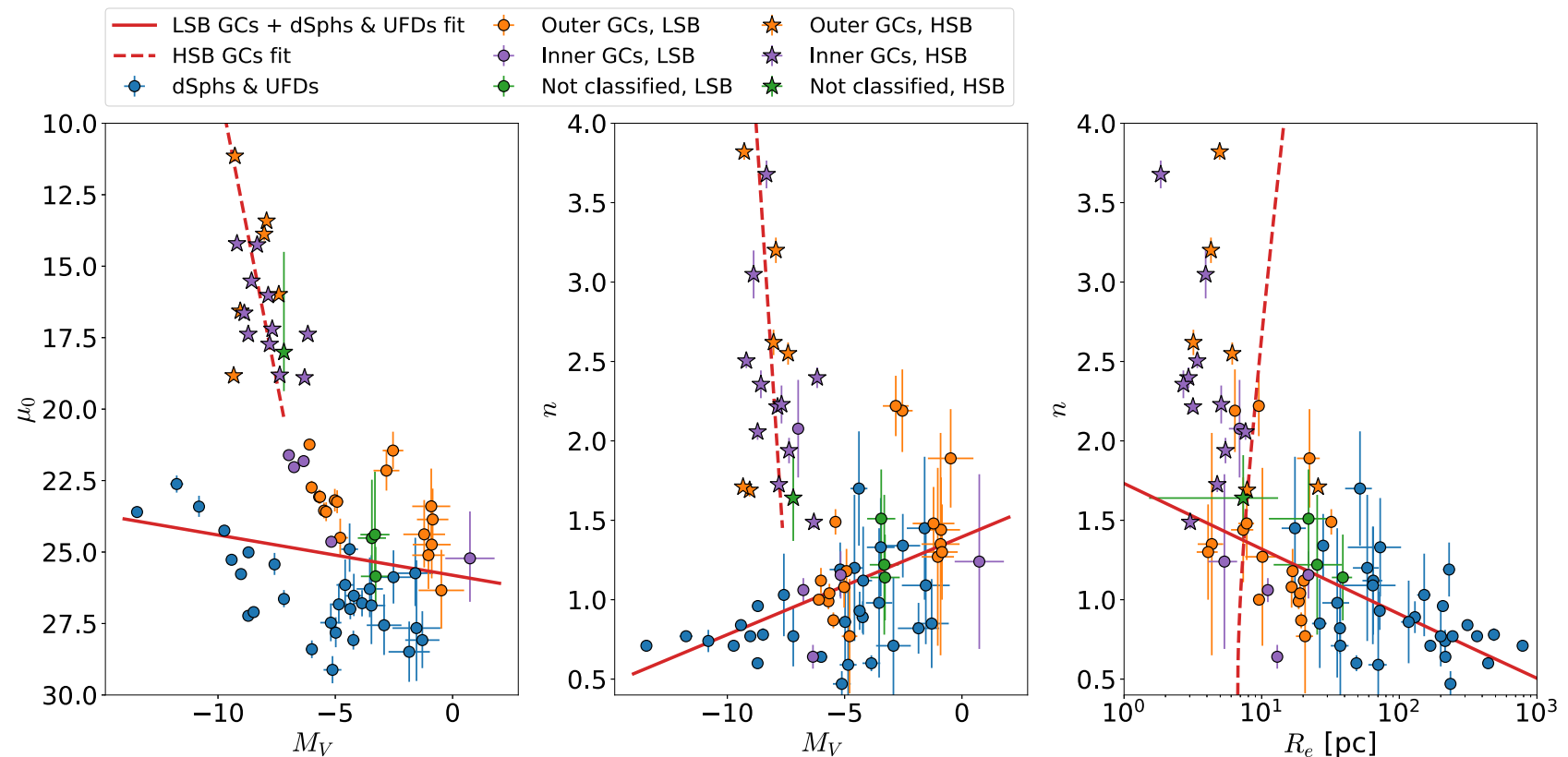

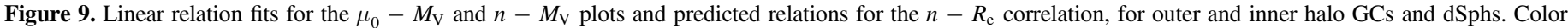

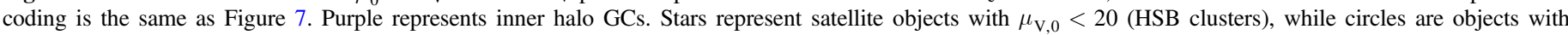

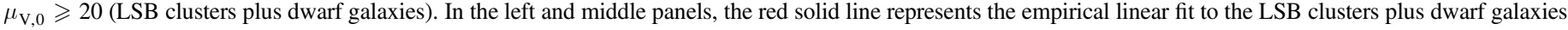

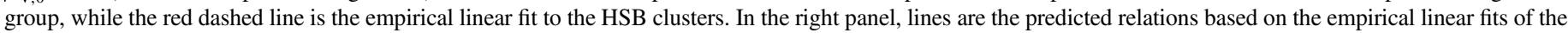
left and right panels.

solid line is the best linear fit to the LSB GC + dwarf galaxy group, while the red dashed line is the best linear fit to the HSB GC group. The right panel shows the predicted relations for the two groups, according to Equation (6). Objects in the LSB GC $+\mathrm{dSph}$ group follow the predicted relation (red solid line). On the other hand, clusters in the HSB group do not follow the relation predicted for them (red dashed line). This is surprising, because the linear fits for the HSB group closely follow the objects' distributions. One possible explanation for this is that HSB clusters are not well described by a pure Sérsic profile and so Equation (3) does not hold for the HSB clusters. Supporting this idea, Figure 8 shows that the Sérsic fit to the radial density profile in most cases is not ideal, especially in the central part, where the Sérsic profile is cuspy and the observed radial density follows a core profile. The same is true for the outer halo GCs in the HSB group (Palomar 2, NGC 2419, NGC 5694, NGC 5824, NGC 6229, and NGC 7006; see Figures 6 to 16 in Muñoz et al. 2018b).

The fact that the $n-R_{\mathrm{e}}$ correlation can be reproduced by using the same empirical relations for the LSB GCs and the $\mathrm{dSphs}$ and that the HSB clusters move off this trend might hint at the existence of two different groups of MW outer halo GCs, one composed of GCs with structural and photometric properties similar to dwarf galaxies, and one composed of some outer halo GCs with properties more similar to inner halo GCs.

\section{6. $D M$ in $G C s$}

The continuity between GCs and dwarf galaxies in the $n-R_{\mathrm{e}}$ plot, together with the overlap of the LSB cluster with ultra-faint galaxies in the $n-M_{\mathrm{V}}$ and $\mu_{0}-M_{\mathrm{V}}$ plots, shows that the photometric properties of an important number of the MW's clusters, at least in this plane, seem indistinguishable from those of dwarf galaxies. This may point to a common formation process for these two types of objects. Because it is commonly accepted that dwarf galaxies are embedded in the DM halo in which they formed, it is perhaps tempting to assume that GCs are also contained and/or were formed inside DM minihalos.

This is not necessarily a controversial idea, because simulations of GCs forming and relaxing inside DM halos do exist, and they reproduce properties observed in real GCs (Mashchenko \& Sills 2005a). Other simulations show that tidal effects of the host galaxy can remove a large amount of the original DM inside GCs (Mashchenko \& Sills 2005b). More recently, Peñarrubia et al. (2017) showed that stars ejected due to hard encounters in the central region of GCs embedded in a DM halo generate an envelope of gravitationally bounded stars. Supporting this model, so-called extratidal stars have been observed in many GCs of the MW (e.g., Carballo-Bello et al. 2012, 2018) and the Andromeda galaxy (Mackey et al. 2010). Additionally, Ibata et al. (2013) concluded that the presence of DM cannot be ruled out from the outer parts of the cluster NGC 2419.

\subsection{Possible Origin of the HSB and LSB GC Groups}

In a scenario where all GCs formed through the same process, naively one could expect a continuity in their photometric properties. The existence of the HSB and LSB groups challenges that notion. Here, we postulate that a possible explanation for the existence of these two groups is the effect of different processes of secular evolution, due to different environments, something that is possible if some GCs formed and evolved inside the MW's potential, while others formed inside external satellite galaxies with weaker potentials and were later stripped from them during the MW's hierarchical accretion stage.

Zinn (1993) studied Galactic clusters and found that they can be classified into three different groups, according to their metallicity and horizontal branch (HB) morphology. There is a 
Table 3

HB Classification for the Clusters in Our Sample that Have that Information in Mackey \& van den Bergh (2005)

\begin{tabular}{lcc}
\hline \hline Object & Surface Brightness Class & HB Class \\
\hline NGC 1261 & HSB & YH \\
NGC 1851 & HSB & OH \\
NGC 1904 & HSB & OH \\
NGC 2298 & HSB & OH \\
NGC 2419 & HSB & OH \\
NGC 4147 & HSB & SG \\
NGC 4590 & HSB & YH \\
NGC 5024 & HSB & OH \\
NGC 5272 & HSB & YH \\
NGC 5634 & HSB & OH \\
NGC 5694 & HSB & OH \\
NGC 5824 & HSB & OH \\
NGC 6229 & HSB & YH \\
NGC 6864 & HSB & OH \\
NGC 7006 & HSB & YH \\
NGC 7078 & HSB & YH \\
Palomar 2 & HSB & YH \\
\hline AM 1 & LSB & YH \\
Eridanus & LSB & YH \\
NGC 5053 & LSB & YH \\
NGC 5466 & LSB & YH \\
NGC 7492 & LSB & OH \\
Palomar 13 & LSB & YH \\
Palomar 14 & LSB & YH \\
Palomar 15 & LSB & OH \\
Palomar 3 & LSB & YH \\
Palomar 4 & LSB & YH \\
Palomar 5 5 LSB & \\
Pyxis & LSB & \\
\hline & & \\
\hline
\end{tabular}

Note. YH stands for young halo cluster, OH for old halo cluster, and SG for the Sagittarius cluster.

metal-rich group $([\mathrm{Fe} / \mathrm{H}]>-0.8)$ located in the bulge and disk of the Galaxy (the bulge/disk group, or BD group), while a more metal-poor group $([\mathrm{Fe} / \mathrm{H}]<-0.8)$ is found in the Galactic halo. Zinn also found that the halo group contains clusters that can have a redder or bluer HB morphology for the same metallicity. This is the known second parameter effect and can be attributed to the age of the clusters, with redder clusters being younger than bluer ones for the same metallicity. This led to the definition of the Old Halo $(\mathrm{OH})$ and Young Halo (YH) groups, where the former formed in situ during a dissipative collapse while the latter formed inside the potential of dwarf galaxies that were later accreted by the MW.

Later, Mackey \& Gilmore (2004) supported this view, showing that the metallicities and HB morphologies of GCs confirmed to be members of the Large Magellanic Cloud, Small Magellanic Cloud, and the Fornax and Sagittarius galaxies are consistent with $\mathrm{YH}$ group values (see their Figure 13).

With this in mind, we can explore whether the classification of GCs into HSB and LSB is consistent with the existence of the $\mathrm{BD}, \mathrm{OH}$, and $\mathrm{YH}$ groups. We use Table 1 of Mackey \& van den Bergh (2005) to obtain the classification in the Zinn scheme for the clusters in our sample. Nine of them, all part of the LSB group, are not listed in the table. Moreover, these clusters do not show any $\mathrm{HB}$ in the color-magnitude plot in Muñoz et al. (2018b), so it is not possible to measure an HB index, which is necessary to classify them (Table 3 shows this classification for our clusters present in Mackey \& van den Bergh 2005). Of the remaining clusters, we count 17 GCs in the HSB group and 13 in the LSB group (in both cases including the inner halo GCs from Carballo-Bello et al. 2012). According to the classification in Mackey \& van den Bergh (2005), of the HSB group, nine clusters are $\mathrm{OH}$, seven are $\mathrm{YH}$, and one is an SG (part of the Sagittarius dwarf galaxy); in the LSB group, two are $\mathrm{OH}$ and 11 are $\mathrm{YH}$. In other words, about half of the clusters in the HSB group are consistent with an in situ origin and the other half are consistent with an external origin, while in the LSB group, the vast majority $(\sim 85 \%)$ are consistent with an external origin.

To explain the current properties of HSB and LSB clusters, tidal stripping processes must have affected GCs differently. In light of the idea that $\mathrm{OH}$ clusters formed in situ and $\mathrm{YH}$ clusters did so in external galaxies, it is evident that HSB and LSB clusters must have been affected by different tidal forces during their secular evolution because they were located at a different galactic host environment. $\mathrm{OH}$ clusters, formed in situ, were subjected to a stronger tidal force, stripping stars from highluminosity clusters (this would give rise to $\mathrm{OH}$ clusters with HSB group characteristics) and completely disintegrating clusters of lower stellar mass (this would explain why there are almost no $\mathrm{OH}$ clusters with LSB group properties). $\mathrm{YH}$ clusters, on the other hand, formed in external galaxies with weaker potential, so they were affected by a weaker tidal force. Later, with the accretion of dwarf galaxies by the MW, they were incorporated into its GC system. Some of these clusters have already been disrupted by the MW's tidal force, leaving a stream of stars behind (e.g., Grillmair 2009); others are in the process of disintegration, as evidenced by the tidal tails emerging from them (e.g., Palomar 5; Rockosi et al. 2002); and others still survive because they have not been affected by the MW's tidal force long enough or they do not live in destructive orbits. Among this last group, there are clusters of low luminosity and extended (the ones that constitute the LSB group) and others of higher luminosity and compact (characteristics of HSB GCs).

Hurley \& Mackey (2010), through $N$-body simulations, provided further insights into the formation of GCs in galactic gravitational potentials of different intensities. They showed that Large Magellanic Cloud-like galaxies of weak tidal fields can produce extended clusters of up to $30 \mathrm{pc}$ from a standard process of formation and evolution. Furthermore, they showed that, for GCs forming in MW-like tidal fields at $10 \mathrm{kpc}$ from the galactic center, their maximum half-light radius is $\sim 10 \mathrm{pc}$. Finally, they pointed out that MW-like galaxies could form extended clusters at large galactocentric distances $(\sim 100 \mathrm{kpc})$ and any extended cluster present at the inner portions of the Galaxy likely formed inside an accreted dwarf galaxy. These simulations support the idea that LSB clusters (typically extended) formed in accreted dwarf galaxies, while HSB clusters (usually more compact) formed inside the MW.

The notion that LSB GCs are of external origin while HSBs are a mix of clusters formed in situ and externally could explain the differences presented in this work. To confirm or reject this idea, the best way is to know the orbit of each satellite object. However, this has proven to be a hard task, because to constrain their orbits, it is necessary to perform high-precision phase space measurements, something that is difficult in objects with a low number of member and/or low-luminosity stars. 
The Gaia mission promises high-precision kinematic information for many of the satellite objects. In fact, the second data release of this mission has already provided us with very accurate proper motions for some satellite dwarf galaxies (Fritz et al. 2018; Gaia Collaboration et al. 2018; Kallivayalil et al. 2018; Massari \& Helmi 2018; Pace \& Li 2018; Simon 2018) and inner halo GCs (Gaia Collaboration et al. 2018; Vasiliev 2019). This new information has allowed us to conclude that most UFDs inside a galactocentric radius of $100 \mathrm{kpc}$ follow eccentric, high-velocity, and retrograde orbits, and some of the galaxies are consistent with being in their first infall (Simon 2018). In the case of GCs, Vasiliev (2019) showed that clusters in $R_{\mathrm{G}} \lesssim 10 \mathrm{kpc}$ rotate in prograde orbits and that the velocity dispersion is isotropic, while for clusters farther out, the velocity distribution becomes radially anisotropic.

In light of these results, we predict that the LSB GCs should follow orbits similar to UFDs' and that they are on their first infall. This last point is consistent with the existence of such low-luminosity, LSB objects inside the strong tidal field of the MW. Also, they should exhibit a radially anisotropic velocity distribution. For the HSB group, given its mixed composition, we predict that GCs located in the inner halo should follow prograde orbits (consistent with clusters formed in situ) with an isotropic velocity dispersion, while the ones located near the frontier between the inner and outer halos should have kinematics similar to LSB clusters.

\section{Conclusions}

In this work, we explored in detail a strong correlation between the Sérsic index and half-light radius that is followed by almost all outer halo satellite objects included in our Megacam sample (Muñoz et al. 2018a). More importantly, in this trend, a large number of GCs follow the same locus as dwarf galaxies, adding support to the similarities between these two types of objects.

We followed the procedure of Graham \& Driver (2005) to see if the correlation in the $n-R_{\mathrm{e}}$ plot can be a consequence of empirical linear relations in the $\mu_{0}-M_{\mathrm{V}}$ and $n-M_{\mathrm{V}}$ parameter spaces for objects that follow a Sérsic density profile. We showed that this is possible if we consider two different classes of outer halo GCs: one that is composed of clusters of LSB, with properties similar to UFD galaxies (the LSB group), and another that is composed of clusters of HSB, with properties similar to inner halo GCs (the HSB group). From our analysis, we saw that empirical linear relations can be fit to the LSB GC + dwarf galaxy group and for the HSB GC group (including the inner GCs). However, for HSB GCs, the $n-R_{\mathrm{e}}$ relation cannot be reproduced, probably because they are not fully described by a pure Sérsic profile.

Given the strong similarities between LSB GCs and dwarf galaxies, and considering that the latter are dominated by DM, we proposed that this is consistent with the notion that GCs also formed inside halos of DM, sharing a common formation process. This idea is supported by previous works that show that GCs with properties similar to what is empirically observed today can be originated by a formation process inside a DM halo.

Finally, to explain the existence of HSB and LSB GCs in a scenario where all clusters formed through a common process, we proposed that tidal effects of the host galaxy play a major role in shaping the cluster's properties. GCs of both types are formed inside MW-like and dwarf galaxies. However, the ones formed inside MW-like galaxies are subjected to stronger tidal forces than the ones inside dwarf galaxies. Thus, LSB GCs inside the MW were quickly disrupted, while HSB GCs, given their higher masses and densities, survived, albeit losing part of their mass; on the other hand, both HSB and LSB GCs survived inside dwarf galaxies. Later, during the process of accretion of dwarf galaxies by the MW, the external HSB and LSB GC populations were incorporated into our Galaxy's cluster system. From this moment, the stronger potential of the MW started its tidal effect over them. The scenario just proposed would explain the observed proportion of external and in situ origin for both HSB and LSB GCs. In fact, following the classification scheme proposed by Zinn (1993), around half of the HSB GCs are of OH type, while the other half is of $\mathrm{YH}$ type, a distribution consistent with a mix of external and in situ origin. In contrast, for the LSB group, almost all of them are of YH type, suggesting that the majority of them were stripped from accreted dwarf galaxies.

Future high-precision proper motion measurements of satellite galaxies, especially for UFDs and outer halo GCs, will allow us to know the true origin of HSB and LSB clusters. We predict that the majority of LSB clusters should have orbits similar to UFDs and dSphs, while HSB clusters should orbit the Galaxy in a way similar to inner halo GCs.

S.M-L. acknowledges support from CONICYT/Doctorado Nacional/2013-21130655. J.A.C-B. acknowledges financial support from CAS-CONICYT 17003. R.R.M. acknowledges partial support from BASAL Project AFB-170002 as well as FONDECYT project $\mathrm{N}^{\circ} 1170364$.

\section{ORCID iDs}

Sebastián Marchi-Lasch (10) https://orcid.org/0000-00024368-9835

Felipe A. Santana (iD https://orcid.org/0000-0002-4023-7649

Marla Geha (iD https://orcid.org/0000-0002-7007-9725

Peter B. Stetson (i) https://orcid.org/0000-0001-6074-6830

S. G. Djorgovski iD https://orcid.org/0000-0002-0603-3087

\section{References}

Baumgardt, H., Côté, P., Hilker, M., et al. 2009, MNRAS, 396, 2051 Bechtol, K., Drlica-Wagner, A., Balbinot, E., et al. 2015, ApJ, 807, 50 Belokurov, V., Irwin, M. J., Koposov, S. E., et al. 2014, MNRAS, 441, 2124 Belokurov, V., Walker, M. G., Evans, N. W., et al. 2008, ApJL, 686, L83 Belokurov, V., Walker, M. G., Evans, N. W., et al. 2009, MNRAS, 397, 1748 Belokurov, V., Walker, M. G., Evans, N. W., et al. 2010, ApJL, 712, L103 Belokurov, V., Zucker, D. B., Evans, N. W., et al. 2006, ApJL, 647, L111 Belokurov, V., Zucker, D. B., Evans, N. W., et al. 2007, ApJ, 654, 897 Bullock, J. S., \& Johnston, K. V. 2005, ApJ, 635, 931

Caon, N., Capaccioli, M., \& D’Onofrio, M. 1993, MNRAS, 265, 1013 Capaccioli, M. 1989, in World of Galaxies (Le Monde des Galaxies), ed. H. G. Corwin Jr. \& L. Bottinelli (New York: Springer), 208

Carballo-Bello, J. A., Gieles, M., Sollima, A., et al. 2012, MNRAS, 419, 14

Carballo-Bello, J. A., Martínez-Delgado, D., Navarrete, C., et al. 2018, MNRAS, 474, 683

Chambers, K. C., Magnier, E. A., Metcalfe, N., et al. 2016, arXiv:1612.05560

Dark Energy Survey Collaboration, Abbott, T., Abdalla, F. B., et al. 2016, MNRAS, 460, 1270

Drlica-Wagner, A., Bechtol, K., Rykoff, E. S., et al. 2015, ApJ, 813, 109

Eggen, O. J., Lynden-Bell, D., \& Sandage, A. R. 1962, ApJ, 136, 748

Foreman-Mackey, D., Hogg, D. W., Lang, D., \& Goodman, J. 2013, PASP, 125,306

Fritz, T. K., Battaglia, G., Pawlowski, M. S., et al. 2018, ApJ, 619, A103

Gaia Collaboration, Helmi, A., van Leeuwen, F., et al. 2018, A\&A, 616, A12 
Graham, A. W. 2011, in EAS Publications Series, How Non-Linear Scaling Relations Unify Dwarf and Giant Elliptical Galaxies, ed. M. Koleva, Ph. Prugniel, \& I. Vauglin (Les Ullis: EDP Sciences), 231

Graham, A. W., \& Driver, S. P. 2005, PASA, 22, 118

Grillmair, C. J. 2009, ApJ, 693, 1118

Harris, W. E. 1996, AJ, 112, 1487

Homma, D., Chiba, M., Okamoto, S., et al. 2018, PASJ, 70, S18

Hurley, J. R., \& Mackey, A. D. 2010, MNRAS, 408, 2353

Hwang, N., Lee, M. G., Lee, J. C., et al. 2011, ApJ, 738, 58

Ibata, R., Nipoti, C., Sollima, A., et al. 2013, MNRAS, 428, 3648

Ivezić, Ž., Beers, T. C., \& Jurić, M. 2012, ARA\&A, 50, 251

Johnston, K. V., Hernquist, L., \& Bolte, M. 1996, ApJ, 465, 278

Kallivayalil, N., Sales, L. V., Zivick, P., et al. 2018, ApJ, 867, 19

Kim, D., Jerjen, H., Mackey, D., Da Costa, G. S., \& Milone, A. P. 2015, ApJL, 804, L44

Koposov, S. E., Belokurov, V., Torrealba, G., \& Evans, N. W. 2015, ApJ, 805,130

Laevens, B. P. M., Martin, N. F., Bernard, E. J., et al. 2015a, ApJ, 813, 44

Laevens, B. P. M., Martin, N. F., Ibata, R. A., et al. 2015b, ApJL, 802, L18

Leaman, R., VandenBerg, D. A., \& Mendel, J. T. 2013, MNRAS, 436, 122

Mackey, A. D., Ferguson, A. M. N., Irwin, M. J., et al. 2010, MNRAS, 401, 533

Mackey, A. D., \& Gilmore, G. F. 2004, MNRAS, 355, 504

Mackey, A. D., \& van den Bergh, S. 2005, MNRAS, 360, 631

Majewski, S. 2004, in Satellites and Tidal Streams, ed. F. Prada,

D. Martinez Delgado, \& T. J. Mahoney (San Francisco, CA: ASP), 63

Martin, N. F., de Jong, J. T. A., \& Rix, H.-W. 2008, ApJ, 684, 1075

Martin, N. F., Nidever, D. L., Besla, G., et al. 2015, ApJL, 804, L5

Mashchenko, S., \& Sills, A. 2005a, ApJ, 619, 243

Mashchenko, S., \& Sills, A. 2005b, ApJ, 619, 258

Massari, D., \& Helmi, A. 2018, arXiv:1805.01839

Mayer, L., Moore, B., Quinn, T., Governato, F., \& Stadel, J. 2002, MNRAS, 336,119

McConnachie, A. W. 2012, AJ, 144, 4

McLaughlin, D. E. 2000, ApJ, 539, 618
Muñoz, R. R., Côté, P., Santana, F. A., et al. 2018a, ApJ, 860, 65

Muñoz, R. R., Côté, P., Santana, F. A., et al. 2018b, ApJ, 860, 66

Muñoz, R. R., Geha, M., Côté, P., et al. 2012a, ApJL, 753, L15

Muñoz, R. R., Padmanabhan, N., \& Geha, M. 2012b, ApJ, 745, 127

Pace, A. B., \& Li, T. S. 2018, arXiv:1806.02345

Peñarrubia, J., Varri, A. L., Breen, P. G., Ferguson, A. M. N., \& Sánchez-Janssen, R. 2017, MNRAS, 471, L31

Rejkuba, M., Dubath, P., Minniti, D., et al. 2007, A\&A, 469, 147

Ripepi, V., Clementini, G., Di Criscienzo, M., et al. 2007, ApJL, 667, L61

Rockosi, C. M., Odenkirchen, M., Grebel, E. K., et al. 2002, AJ, 124, 349

Sand, D. J., Seth, A., Olszewski, E. W., et al. 2010, ApJ, 718, 530

Searle, L., \& Zinn, R. 1978, ApJ, 225, 357

Sérsic, J. L. 1968, Atlas de Galaxias Australes (Córdoba: Observatorio Astronómico)

Simon, J. D. 2018, ApJ, 863, 89

Torrealba, G., Koposov, S. E., Belokurov, V., et al. 2016a, MNRAS, 463,712

Torrealba, G., Koposov, S. E., Belokurov, V., \& Irwin, M. 2016b, MNRAS, 459,2370

Trujillo, I., Graham, A. W., \& Caon, N. 2001, MNRAS, 326, 869

van den Bergh, S., \& Mackey, A. D. 2004, MNRAS, 354, 713

Vasiliev, E. 2019, MNRAS, 484, 2832

Walsh, S. M., Jerjen, H., \& Willman, B. 2007, ApJL, 662, L83

Willman, B. 2010, AdAst, 2010, 285454

Willman, B., Dalcanton, J. J., Martinez-Delgado, D., et al. 2005, ApJL, 626, L85

Willman, B., \& Strader, J. 2012, AJ, 144, 76

York, D. G., Adelman, J., Anderson, J. E., Jr., et al. 2000, AJ, 120, 1579

Zaritsky, D., Crnojević, D., \& Sand, D. J. 2016, ApJ, 826, L9

Zinn, R. 1993, in The Globular Cluster-Galaxy Connection, ed. G. H. Smith \& J. P. Brodie (San Francisco, CA: ASP), 38

Zinn, R. 1996, in Formation of the Galactic Halo...Inside and Out, ed. H. L. Morrison \& A. Sarajedini (San Francisco, CA: ASP), 211

Zucker, D. B., Belokurov, V., Evans, N. W., et al. 2006, ApJL, 650, L41 